\title{
The category of Colombeau algebras
}

\author{
Lorenzo Luperi Baglini $^{1}$ • Paolo Giordano ${ }^{1}$
}

Received: 27 October 2015 / Accepted: 4 October 2016 / Published online: 11 October 2016 C The Author(s) 2016. This article is published with open access at Springerlink.com

\begin{abstract}
Since the beginning of Colombeau's the theory of algebras of generalized functions, the role of its characteristic polynomial growth versus a more general condition has been explored. Recently, we introduced the notion of asymptotic gauge (AG), and we used it to study Colombeau AG-algebras. This construction concurrently generalizes many different algebras used in Colombeau's theory and, at the same time, allows for more general growth scales. In this paper, we study the categorical properties of Colombeau AG-algebras with respect to the choice of the AG. The main aim of the paper is to study suitable functors to relate differential equations framed in algebras having different growth scales.
\end{abstract}

Keywords Colombeau-type algebras · Functorial properties · Asymptotic gauges · Embeddings of distributions $\cdot$ Sets of indices

Mathematics Subject Classification Principal 46F30; Secondary 18B99

Communicated by A. Constantin.

P. Giordano has been supported by Grants P25116-N25 and P25311-N25 of the Austrian Science Fund FWF. Luperi Baglini has been supported by Grants P25311-N25 and M1876-N35 of the Austrian Science Fund FWF.

$凶$ Lorenzo Luperi Baglini

lorenzo.luperi.baglini@univie.ac.at

Paolo Giordano

paolo.giordano@univie.ac.at

1 Faculty of Mathematics, University of Vienna, Oskar-Morgenstern-Platz 1, 1090 Vienna, Austria 


\section{Introduction}

Colombeau algebras are algebras of generalized functions introduced by Colombeau in the early 1980s in order to rigorously define multiplication and other nonlinear operations on Schwartz distributions, see [1-3,5]. Containing the space of Schwartz distributions as a linear subspace and the algebra of smooth functions as a subalgebra, they permit to bypass the Schwartz impossibility result. This impossibility result, see [20], states that whenever the space $\mathcal{D}^{\prime}(\Omega)$ of (real valued) distributions on the open set $\Omega \subseteq \mathbb{R}^{n}$ is embedded into an associative differential algebra $\mathcal{G}(\Omega)$, the latter cannot at the same time extend the distributional derivatives and the pointwise product of continuous functions. In particular, if $\mathcal{T} \mathbb{R}^{n}$ is the usual Euclidean topology on $\mathbb{R}^{n}$, Colombeau's construction resulted in the following remarkable properties:

(a) $\Omega \in\left(\mathcal{T} \mathbb{R}^{n}\right)^{\text {op }} \mapsto \mathcal{G}(\Omega)$ is a presheaf of commutative and associative differential algebras with respect to suitable derivations $\partial_{\Omega}^{\alpha}: \mathcal{G}(\Omega) \longrightarrow \mathcal{G}(\Omega)$ for all $\alpha \in \mathbb{N}^{n}$.

(b) The set of generalized scalars $\widetilde{\mathbb{R}}=\left\{f \in \mathcal{G}(\mathbb{R}) \mid \partial_{\mathbb{R}}^{\alpha} f=0 \forall \alpha\right\}$ is a nonArchimedean ring (with zero divisors).

(c) The embedding $\iota_{\Omega}: \mathcal{D}^{\prime} \longrightarrow \mathcal{G}$ is a natural transformation between sheaves of real vector spaces.

(d) The derivations are compatible, i.e. if $D_{\Omega}^{\alpha}: \mathcal{D}^{\prime}(\Omega) \longrightarrow \mathcal{D}^{\prime}(\Omega)$ is the usual $\alpha$ derivation of distributions, then $\partial_{\Omega}^{\alpha}\left(\iota_{\Omega}(T)\right)=\iota_{\Omega}\left(D_{\Omega}^{\alpha}(T)\right)$ for all $T \in \mathcal{D}^{\prime}(\Omega)$.

(e) Let $S_{\Omega}: \mathcal{C}^{\infty}(\Omega) \longrightarrow \mathcal{D}^{\prime}(\Omega),\left\langle S_{\Omega}(f), \varphi\right\rangle:=\int_{\Omega} f \varphi$, be the usual Schwartz embedding of smooth functions. Then $\iota_{\Omega}\left[S_{\Omega}\left(\mathcal{C}^{\infty}(\Omega)\right]\right.$ is a differential subring of $\mathcal{G}(\Omega)$ and is naturally isomorphic to $\mathcal{C}^{\infty}(\Omega)$. This natural identification $\mathcal{C}^{\infty}(\Omega)=$ $\iota_{\Omega}\left[S_{\Omega}\left(\mathcal{C}^{\infty}(\Omega)\right] \subseteq \mathcal{G}(\Omega)\right.$ can also be understood as a suitable universal property.

Since Colombeau's original construction, many other differential algebras satisfying properties (a)-(e) have been constructed by Colombeau himself and other authors, see e.g. $[3,5,10,11]$. We also recall that every algebra of Colombeau's type has a non-standard counterpart (see e.g. [17,23,24]). In particular, the non-standard algebra of asymptotic functions $\hat{\mathcal{G}}$, besides satisfying properties (a)-(e), presents better formal properties: its definition involves a reduced number of quantifiers, the ring $\widetilde{\mathbb{R}}$ is a real closed Cantor complete field, the field of complex generalized scalars $\widetilde{\mathbb{C}}=\widetilde{\mathbb{R}}(\sqrt{-1})$ is algebraically closed, and a Hahn-Banach extension principle holds, see e.g. [23]. For the analogue of $\widetilde{\mathbb{R}}$ in non-standard analysis (NSA), see [16,19,23].

The relevance of properties (a)-(e) can also be highlighted by mentioning that they are included in the axiomatic approach of [21,22].

Due to its relative simplicity, the most studied Colombeau-like algebra is surely the special one, which is the quotient algebra $\mathcal{G}^{s}(\Omega):=\mathcal{E}_{M}(\Omega) / \mathcal{N}^{s}(\Omega)$, where ${ }^{1}$

$$
\begin{aligned}
& \mathcal{E}_{M}(\Omega):=\left\{\left(u_{\varepsilon}\right) \in \mathcal{C}^{\infty}(\Omega)^{(0,1]}\left|\forall K \Subset \Omega \forall \alpha \in \mathbb{N}^{n} \exists N \in \mathbb{N}: \sup _{x \in K}\right| \partial^{\alpha} u_{\varepsilon}(x) \mid=O\left(\varepsilon^{-N}\right)\right\} \\
& \mathcal{N}^{s}(\Omega):=\left\{\left(u_{\varepsilon}\right) \in \mathcal{C}^{\infty}(\Omega)^{(0,1]}\left|\forall K \Subset \Omega \forall \alpha \in \mathbb{N}^{n} \forall m \in \mathbb{N}: \sup _{x \in K}\right| \partial^{\alpha} u_{\varepsilon}(x) \mid=O\left(\varepsilon^{m}\right)\right\} .
\end{aligned}
$$

1 In the naturals $\mathbb{N}=\{0,1,2,3 \ldots\}$ we include zero. 
Although Colombeau algebras were essentially introduced to find solutions of nonlinear differential equations which are not solvable in classical spaces of distributions, it is well known that trivial $\widetilde{\mathbb{R}}$-linear ODEs remain not solvable also in these algebras, like e.g.

$$
\left\{\begin{array}{l}
x^{\prime}(t)=\left[\varepsilon^{-1}\right] \cdot x(t) \\
x(0)=1
\end{array}\right.
$$

(see also [5,12]). A step towards the analysis of these problems is the generalization of the role of the infinite nets $\left(\varepsilon^{-n}\right) \in \mathbb{R}^{(0,1]}, n \in \mathbb{N}$, appearing in the definition of Colombeau algebras, see (1.1). An understanding of the role of the nets $\left(\varepsilon^{-n}\right) \in \mathbb{R}^{(0,1]}$ has already been realized through the notions of asymptotic scales, $(\mathcal{C}, \mathcal{E}, \mathcal{P})$-algebras, $\left(\mathcal{M}, \mathcal{N}, V_{\mathcal{P}}\right)$-algebras, exponent weights and asymptotic gauges, see $[6-9,12,14,15]$ and references therein.

In particular, if one considers the usual sheaf of smooth functions $\mathcal{C}^{\infty}(-)$, Colombeau AG-algebras are among the simplest and most general approaches (only [14] is actually more general). In fact, Colombeau AG-algebras include in the same abstract framework all known Colombeau-like algebras, like the special one $\mathcal{G}^{\text {s }}$, the full one $\mathcal{G}^{\mathrm{e}}$, [5], the NSA based algebra of asymptotic functions $\hat{\mathcal{G}}$, [23], the diffeomorphism invariant algebras $\mathcal{G}^{\mathrm{d}}, \mathcal{G}^{2}$ and $\hat{\mathcal{G}}$, [5], the Egorov algebra, [10,11,25], and the algebra of non-standard smooth functions ${ }^{*} \mathcal{C}^{\infty}(\Omega)$, $[4,18]$; see $[12,13]$ and below. The simplicity of the approach with AGs lies, for all the algebras mentioned above, in the use of the simple logical structure of quantifiers that characterizes the special algebra $\mathcal{G}^{\mathrm{s}}$. In order to establish a conceptual knowledge of this multiplicity of differential algebras, the abstract framework of Colombeau AG-algebras is therefore a step towards the understanding of the core properties of Colombeau's construction.

Considering different scales instead of the polynomial one $\mathcal{B}_{\text {pol }}:=\left\{\left(\varepsilon^{-n}\right) \mid n \in \mathbb{N}\right\}$ occurring in (1.1), we can e.g. take

$$
\mathcal{B}_{\exp }:=\left\{\left(e^{n / \varepsilon}\right) \mid n \in \mathbb{N}\right\} \quad \text { or } \quad \mathcal{B}_{\exp ^{2}}:=\left\{\left(e^{e^{n / \varepsilon}}\right) \mid n \in \mathbb{N}\right\}
$$

to obtain two different Colombeau AG-algebras $\mathcal{G}^{s}\left(\Omega, \mathcal{B}_{\exp }\right)$ and $\mathcal{G}^{s}\left(\Omega, \mathcal{B}_{\exp ^{2}}\right)$. In both these algebras, we can solve the ODE (1.2), which is not solvable in the classical special algebra $\mathcal{G}^{s}(\Omega)=\mathcal{G}^{s}\left(\Omega, \mathcal{B}_{\text {pol }}\right)$. But these solutions $x_{1} \in \mathcal{G}^{s}\left(\Omega, \mathcal{B}_{\text {exp }}\right)$ and $x_{2} \in \mathcal{G}^{s}\left(\Omega, \mathcal{B}_{\exp ^{2}}\right)$ of (1.2) belong to different quotient algebras, and it is hence natural to investigate how to relate them. The best way to solve this kind of problems is to study the categorical properties of Colombeau AG-algebras $\mathcal{G}^{s}(\Omega, \mathcal{B})$ with respect to a change of the AG $\mathcal{B}$. In fact, the right concept of morphism of AGs permits to connect $\mathcal{G}^{s}\left(\Omega, \mathcal{B}_{\exp }\right)$ and $\mathcal{G}^{s}\left(\Omega, \mathcal{B}_{\exp ^{2}}\right)$ using the functorial properties of $\mathcal{G}^{s}(\Omega,-)$. We shall frequently use (1.2) as a paradigmatic motivation, even if the solution of this trivial ODE is not one of the aim of the present paper. For the solution and uniqueness of all $\widetilde{\mathbb{R}}$-linear ODE in a minimal Colombeau AG-algebra, see [12].

In the context of AGs, it is therefore natural to set the following questions as main motivations of the present paper: 
- Is the construction of the Colombeau algebra $\mathcal{G}(\Omega, \mathcal{B})$ functorial with respect to the AG $\mathcal{B}$ ? Is this construction functorial with respect to the open set $\Omega$ ?

- When can we consider two AGs as isomorphic? For instance, we will show that the $\mathrm{AG}$ of polynomial growth $\mathcal{B}_{\text {pol }}$ is isomorphic to the $\mathrm{AG}$ of exponential growth $\mathcal{B}_{\text {exp }}$. This isomorphism holds in spite of the fact that using the latter we can solve ODE which are not solvable with the former, see Sect. 7.

- How to relate the solutions of differential equations framed in a given Colombeau AG-algebra to those framed into another one?

- Colombeau theory can be more clearly summarized by saying that it permits to define a differential algebra together with an embedding of Schwartz's distributions. This embedding can be intrinsic, or diffeomorphism invariant, or it can be chosen in order to have properties like $H(0)=\frac{1}{2}$, where $H$ is the Heaviside's step function. Can we define a general category of Colombeau algebras having as objects triples $(G, \partial, \iota)$ made of an algebra $G$, a family of derivations $\partial$ and an embedding of distributions $\iota$ ? Can we see $\mathcal{G}^{\text {s }}$ as a suitable functor with values in this category? What is the domain of this functor?

- Similarly to the axiomatic approach of [21,22], in defining this category of Colombeau algebras, we need to focus on peculiar properties of Colombeau-like algebras. This approach represents another way to establish a certain order of importance in the properties satisfied by all algebras of Colombeau type.

In the present work, we answer these questions using basic category theory and the abstract language of AGs as a unifying framework. In this way, we are going to solve these problems for each one of the aforementioned Colombeau-like algebras. The paper assumes a certain knowledge of Colombeau theory, even if it is completely self-contained concerning concepts related to AGs.

\section{Sets of indices}

\subsection{Basic definitions}

In [13], the general notion of sets of indices has been introduced. For reader's convenience, in this section we recall the notations and notions from [13] that we will use in the present work. For all the proofs, we refer to [13].

Definition 1 We say that $\mathbb{I}=(I, \leq, \mathcal{I})$ is a set of indices if the following conditions hold:

(i) $(I, \leq)$ is a pre-ordered set, i.e., $I$ is a non empty set with a reflexive and transitive relation $\leq$;

(ii) $\mathcal{I}$ is a set of subsets of $I$ such that $\emptyset \notin \mathcal{I}$ and $I \in \mathcal{I}$;

(iii) $\forall A, B \in \mathcal{I} \exists C \in \mathcal{I}: C \subseteq A \cap B$.

For all $e \in I$, set $(\emptyset, e]:=\{\varepsilon \in I \mid \varepsilon \leq e\}$. As usual, we say $\varepsilon<e$ if $\varepsilon \leq e$ and $\varepsilon \neq e$. Using these notations, we state the last condition in the definition of set of indices:

(iv) If $e \leq a \in A \in \mathcal{I}$, the set $A_{\leq e}:=(\emptyset, e] \cap A$ is downward directed by $<$, i.e., it is non empty and $\forall b, c \in A_{\leq e} \exists d \in A_{\leq e}: d<b, d<c$. 
The following are examples of sets of indices.

Example 2 (i) Let $I^{\mathrm{s}}:=(0,1] \subseteq \mathbb{R}$, let $\leq$ be the usual order relation on $\mathbb{R}$, and let $\mathcal{I}^{\mathrm{s}}:=\left\{\left(0, \varepsilon_{0}\right] \mid \varepsilon_{0} \in I\right\}$. Following [13], we denote by $\mathbb{I}^{\mathrm{s}}:=\left(I^{\mathrm{s}}, \leq, \mathcal{I}^{\mathrm{s}}\right)$ this set of indices.

(ii) If $\varphi \in \mathcal{D}\left(\mathbb{R}^{n}\right), r \in \mathbb{R}_{>0}$ and $x \in \mathbb{R}^{n}$, we use the symbol $r \odot \varphi$ to denote the function $x \in \mathbb{R}^{n} \mapsto \frac{1}{r^{n}} \cdot \varphi\left(\frac{x}{r}\right) \in \mathbb{R}$, see [13]. With the usual notations of [5], we define $I^{\mathrm{e}}:=\mathcal{A}_{0}, \mathcal{I}^{\mathrm{e}}:=\left\{\mathcal{A}_{q} \mid q \in \mathbb{N}\right\}$, and for $\varepsilon, e \in I^{\mathrm{e}}$, we set $\varepsilon \leq e$ iff there exists $r \in \mathbb{R}_{>0}$ such that $r \leq 1$ and $\varepsilon=r \odot e$. Then $\mathbb{I}^{\mathrm{e}}:=\left(I^{\mathrm{e}}, \leq, \mathcal{I}^{\mathrm{e}}\right)$ is a set of indices used in this framework to unify and simplify the full algebra $\mathcal{G}^{\text {e }}$ (see [13, Sect. 3]).

(iii) Let $I^{\mathrm{E}}:=\mathbb{N}$ with the inverse $\lesssim$ of the usual order notion on $\mathbb{N}$ (namely, $m \lesssim n$ iff $m \geq n$ ), and let $\mathcal{I}^{\mathrm{E}}$ be the Fréchet filter of cofinite sets. The set of indices $\mathbb{I}^{\mathrm{E}}:=\left(I^{\mathrm{E}}, \lesssim, \mathcal{I}^{\mathrm{E}}\right)$ is used by $[10,11]$ to introduce the so-called Egorov algebra. The analogue in NSA is $\mathbb{I}^{*}:=\left(\mathbb{N}, \lesssim, \mathcal{I}^{*}\right)$, where $\mathcal{I}^{*}$ is a free ultrafilter that contains $\mathcal{I}^{\mathrm{E}}$. The set of indices $\mathbb{I}^{*}$ is used to define the algebra of non-standard smooth functions ${ }^{*} \mathcal{C}^{\infty}(\Omega)$, see [18].

(iv) For every $\varphi \in \mathcal{A}_{0}$, let us call order of $\varphi$ the natural number

$$
o(\varphi):=\min \left\{n \in \mathbb{N} \mid \varphi \in \mathcal{A}_{n} \backslash \mathcal{A}_{n+1}\right\}
$$

and, for every $\varphi, \psi \in \mathcal{A}_{0}$, set

$$
\varphi \lesssim \psi \quad \text { iff } o(\varphi)<o(\psi) \text { or } \varphi \leq \psi \text { in } \mathbb{I}^{\mathrm{e}} .
$$

We have that $\widetilde{\mathbb{I}}^{\mathrm{e}}=\left(\mathcal{A}_{0}\right.$, $\left.\lesssim,\left\{\mathcal{A}_{q} \mid q \in \mathbb{N}\right\}\right)$ is a downward directed set of indices that can be used to try a simplification of the full algebra $\mathcal{G}^{\mathrm{e}}$. See Sect. 3.1 for the nicer properties that downward directed sets have with respect to the notions we are going to introduce.

Henceforward, functions of the type $f: I \longrightarrow \mathbb{R}$ are called nets, and for their evaluation we both use the notations $f_{\varepsilon}$ or $f(\varepsilon)$, the latter in case the subscript notation were too cumbersome. When the domain $I$ is clear, we use also the notation $f=\left(f_{\varepsilon}\right)$ for the whole net. Analogous notations will be used for nets of smooth functions $u=\left(u_{\varepsilon}\right) \in \mathcal{C}^{\infty}(\Omega)^{I}$.

In each set of indices, we can define two notions of big-O for nets of real numbers. These two big-Os share the same (usual) properties of the classical one as preorders and concerning algebraic operations (see [13, Thm. 2.8, Thm. 2.14]). Since each set of the form $A_{\leq e}=(\emptyset, e] \cap A$ is downward directed, the first big-O is the usual one:

Definition 3 Let $\mathbb{I}=(I, \leq, \mathcal{I})$ be a set of indices. Let $a \in A \in \mathcal{I}$ and let $\left(x_{\varepsilon}\right)$, $\left(y_{\varepsilon}\right) \in \mathbb{R}^{I}$ be two nets of real numbers defined in $I$. We write

$$
x_{\varepsilon}=O_{a, A}\left(y_{\varepsilon}\right) \quad \text { as } \varepsilon \in \mathbb{I}
$$

if

$$
\exists H \in \mathbb{R}_{>0} \exists \varepsilon_{0} \in A_{\leq a} \forall \varepsilon \in A_{\leq \varepsilon_{0}}:\left|x_{\varepsilon}\right| \leq H \cdot\left|y_{\varepsilon}\right| .
$$


The second notion of big-O is the following:

Definition 4 Let $\mathbb{I}=(I, \leq \mathcal{I})$ be a set of indices. Let $\mathcal{J} \subseteq \mathcal{I}$ be a non empty subset of $\mathcal{I}$ such that

$$
\forall A, B \in \mathcal{J} \exists C \in \mathcal{J}: C \subseteq A \cap B .
$$

Finally, let $\left(x_{\varepsilon}\right),\left(y_{\varepsilon}\right) \in \mathbb{R}^{I}$ be nets of real numbers. Then we say

$$
x_{\varepsilon}=O_{\mathcal{J}}\left(y_{\varepsilon}\right) \text { as } \varepsilon \in \mathbb{I}
$$

if

$$
\exists A \in \mathcal{J} \forall a \in A: x_{\varepsilon}=O_{a, A}\left(y_{\varepsilon}\right) .
$$

We simply write $x_{\varepsilon}=O\left(y_{\varepsilon}\right)($ as $\varepsilon \in \mathbb{I})$ when $\mathcal{J}=\mathcal{I}$, i.e. to denote $x_{\varepsilon}=O_{\mathcal{I}}\left(y_{\varepsilon}\right)$.

For example, in the case of the set of indices $\mathbb{H}^{\mathrm{e}}$ used for the full algebra, we have $x_{\varepsilon}=O\left(y_{\varepsilon}\right)$ as $\varepsilon \in \mathbb{I}^{\mathrm{e}}$ if and only if $\exists q \in \mathbb{N} \forall \varphi \in \mathcal{A}_{q}: x(\varepsilon \odot \varphi)=O[y(\varepsilon \odot \varphi)]$ as $\varepsilon \rightarrow 0^{+}$, where the latter big-O is the classical one, see [13, Thm. 3.2]. We can hence recognise an important part of the usual definition of moderate and negligible nets for the full algebra $\mathcal{G}^{\mathrm{e}}$. The abstract approach we use in this paper can be easily understood by interpreting $\mathbb{I}$ in the simplest case $\mathbb{I}^{\mathrm{S}}$ of the special algebra and in the case $\mathbb{I}^{\mathrm{e}}$ of the full algebra. In the former, any formula of the form $\exists A \in \mathcal{I} \forall a \in A$ becomes $\exists \varepsilon_{0} \in(0,1] \forall \varepsilon \in\left(0, \varepsilon_{0}\right]$. In the latter it becomes $\exists q \in \mathbb{N} \forall \varphi \in \mathcal{A}_{q}$.

In every set of indices we can formalize the notion of for $\varepsilon$ sufficiently small as follows.

Definition 5 Let $\mathbb{I}=(I, \leq, \mathcal{I})$ be a set of indices. Let $a \in A \in \mathcal{I}$ and $\mathcal{P}(-)$ be a property, then we say

$$
\forall^{\mathbb{I}} \varepsilon \in A_{\leq a}: \mathcal{P}(\varepsilon)
$$

and we read it for $\varepsilon$ sufficiently small in $A_{\leq a}$ the property $\mathcal{P}(\varepsilon)$ holds, if

$$
\exists e \in A_{\leq a} \forall \varepsilon \in A_{\leq e}: \mathcal{P}(\varepsilon)
$$

Note that, by condition (iv) of Definition 1, it follows that $A_{\leq e} \neq \emptyset$, so that (2.4) is equivalent to

$$
\exists e \leq a \forall \varepsilon \in A_{\leq e}: \mathcal{P}(\varepsilon)
$$

Moreover, we say that

$$
\forall^{\mathbb{I}} \varepsilon: \mathcal{P}(\varepsilon)
$$

and we read it for $\varepsilon$ sufficiently small in $\mathbb{I}$ the property $\mathcal{P}(\varepsilon)$ holds, if $\exists A \in \mathcal{I} \forall a \in$ $A \forall \forall^{\mathbb{I}} \varepsilon \in A_{\leq a}: \mathcal{P}(\varepsilon)$. 
Using this notion, we can define an order relation for nets.

Definition 6 Let $\mathbb{I}=(I, \leq, \mathcal{I})$ be a set of indices, and $i, j: I \longrightarrow \mathbb{R}$ be nets. Then we say $i>\mathbb{I} j$ if

$$
\forall^{\mathbb{I}} \varepsilon: i_{\varepsilon}>j_{\varepsilon}
$$

Finally, we recall the notion of limit of a net of real numbers:

Definition 7 Let $\mathbb{I}=(I, \leq, \mathcal{I})$ be a set of indices, $f: I \longrightarrow \mathbb{R}$ a map, and $l \in$ $\mathbb{R} \cup\{+\infty,-\infty\}$. Then we say that $l$ is the limit of $f$ in $\mathbb{I}$ if

$$
\exists A \in \mathcal{I} \forall a \in A: l=\left.\lim _{\varepsilon \leq a} f\right|_{A}(\varepsilon),
$$

where the limit (2.5) is taken in the downward directed set $(\emptyset, a]=I_{\leq a}$.

Let us observe that if $l=\left.\lim _{\varepsilon \leq a} f\right|_{A}(\varepsilon)$ and $B \subseteq A, B \in \mathcal{I}$, then $l=\left.\lim _{\varepsilon \leq a} f\right|_{B}(\varepsilon)$; moreover, there exists at most one $l$ verifying (2.5).

\section{The category Ind}

We start by defining the notion of morphism between two sets of indices. This is also a natural step to define the concept of morphism of asymptotic gauges. A natural property to expect from a morphism $f: \mathbb{I}_{1} \longrightarrow \mathbb{I}_{2}$ between sets of indices $\mathbb{I}_{1}, \mathbb{I}_{2}$ is the preservation of the notion of "eventually" for properties $\mathcal{P}$, i.e. that $\forall^{\mathbb{I}_{1}} \varepsilon_{1} \mathcal{P}\left(\varepsilon_{1}\right)$ implies $\forall^{\mathbb{I}_{2}} \varepsilon_{2} \mathcal{P}\left(f\left(\varepsilon_{2}\right)\right)$. Let us note that we start from a property $\mathcal{P}\left(\varepsilon_{1}\right)$, for $\varepsilon_{1} \in I_{1}$, and we want to arrive at a property $\mathcal{P}\left(f\left(\varepsilon_{2}\right)\right)$, for $\varepsilon_{2} \in I_{2}$.

Definition 8 Let $\mathbb{I}_{k}=\left(I_{k}, \leq_{k}, \mathcal{I}_{k}\right)$ be sets of indices for $k=1,2$. Let $a \in A \in$ $\mathcal{I}_{1}, b \in B \in \mathcal{I}_{2}$. Then we say that $f: A_{\leq a} \longrightarrow B_{\leq b}$ is infinitesimal if

(i) $f: I_{2} \longrightarrow I_{1}$;

(ii) $\forall \alpha \in A_{\leq a} \forall^{\mathbb{I}_{2}} \varepsilon_{2} \in B_{\leq b}: f\left(\varepsilon_{2}\right) \in A_{\leq \alpha}$.

Moreover, we say that $f: \mathbb{I}_{1} \longrightarrow \mathbb{I}_{2}$ is a morphism of sets of indices if

$$
\forall A \in \mathcal{I}_{1} \forall a \in A \exists B \in \mathcal{I}_{2} \forall b \in B: f: A_{\leq a} \longrightarrow B_{\leq b} \text { is infinitesimal. }
$$

Therefore, a morphism $f: \mathbb{I}_{1} \longrightarrow \mathbb{I}_{2}$ is a map in the opposite direction $f: I_{2} \longrightarrow I_{1}$ between the underlying sets. Only in this way we have that the map $f$ preserves the asymptotic relations that hold in $\mathbb{I}_{1}$, see Corollary 13 for a list of examples.

Example 9 (i) For every set of indices $\mathbb{I}=(I, \leq, \mathcal{I})$ if $1_{\mathbb{I}}: I \longrightarrow I$ is the identity function then $1_{\mathbb{I}}: \mathbb{I} \longrightarrow \mathbb{I}$ is a morphism.

(ii) Let $f:(0,1] \longrightarrow(0,1]$ be a map, then $f: \mathbb{I}^{\mathrm{s}} \longrightarrow \mathbb{I}^{\mathrm{s}}$ is a morphism if and only if $\forall \varepsilon \in(0,1] \exists \delta \in(0,1]: f((0, \delta]) \subseteq(0, \varepsilon]$, i.e. if and only if $\lim _{\varepsilon \rightarrow 0^{+}} f(\varepsilon)=0$. 
(iii) For the set of indices $\mathbb{I}^{\mathrm{e}}$ of the full algebra, we recall that $\left(\mathcal{A}_{q}\right)_{\leq \varphi}=(\emptyset, \varphi]$ and $\varphi:=\min \{\operatorname{diam}(\operatorname{supp} \varphi), 1\}$. If $f: I^{\mathrm{e}} \longrightarrow I^{\mathrm{e}}$ is a map, then we have that $f: \overline{(\varnothing,} \varphi] \longrightarrow(\emptyset, \psi]$ is infinitesimal if and only if $\forall \varepsilon \in(0,1] \exists \delta \in(0,1]$ : $f(\{r \odot \psi \mid r \in(0, \delta]\}) \subseteq\{r \odot \varphi \mid r \in(0, \varepsilon]\}$. Therefore, this implies that $\lim _{\varepsilon \rightarrow 0^{+}} \underline{f(\varepsilon \odot \psi)}=0$. If we denote by $\frac{f(\varepsilon \odot \psi)}{\varphi}$ the unique $r \in(0,1]$ such that $f(\varepsilon \odot \psi)=r \odot \varphi$ (in case it exists), then $f:(\emptyset, \varphi] \longrightarrow(\emptyset, \psi]$ is infinitesimal if and only if $\lim _{\varepsilon \rightarrow 0^{+}} \frac{f(\varepsilon \odot \psi)}{\varphi}=0$. Moreover, $f: \mathbb{I}^{\mathrm{e}} \longrightarrow \mathbb{I}^{\mathrm{e}}$ is a morphism if and only if $\forall m \in \mathbb{N} \forall \varphi \in \mathcal{A}_{m} \exists q \in \mathbb{N} \forall \psi \in \mathcal{A}_{q}: \lim _{\varepsilon \rightarrow 0^{+}} \frac{f(\varepsilon \odot \psi)}{\varphi}=0$. This and the previous example justify our use of the name infinitesimal in Definition 8.

(iv) Let $\varphi \in \mathcal{A}_{0}$ be fixed, let $\mathbb{I}_{\varphi}:=((\varnothing, \varphi], \leq,\{(\varnothing, \varphi]\})$, where the order relation on $\mathbb{I}_{\varphi}$ is the restriction of the order relation on $\mathbb{I}^{\mathrm{e}}$. If $f:(0,1] \longrightarrow(\emptyset, \varphi]$ is the function $f(r):=r \odot \varphi$ for every $r \in(0,1]$ then we have that $f: \mathbb{I}_{\varphi} \longrightarrow \mathbb{I}^{\mathrm{s}}$ is a morphism. Conversely, if $g:(\emptyset, \varphi] \longrightarrow(0,1]$ maps every $\psi \in(\emptyset, \varphi]$ to the unique $r \in(0,1]$ such that $\psi=r \odot \varphi$, i.e. $g(\psi)=\frac{\psi}{\varphi}$, then $g: \mathbb{I}^{\mathrm{s}} \longrightarrow \mathbb{I}_{\varphi}$ is a morphism. We have that $f=g^{-1}$.

(v) Let $f: \mathbb{N} \longrightarrow \mathbb{N}$ be a map, then $f: \mathbb{I}^{\mathrm{E}} \longrightarrow \mathbb{I}^{\mathrm{E}}$ is a morphism if and only if $\lim _{n \rightarrow+\infty} f(n)=+\infty$. Analogously, $f: \mathbb{I}^{*} \longrightarrow \mathbb{I}^{*}$ is a morphism if and only if there exists an ultrafilter set $B \in \mathcal{I}^{*}$ such that $\lim _{n \rightarrow+\infty} f(n)=+\infty$.

(vi) Let us denote by $\overline{\mathbb{N}}$ the set of indices $\left(\mathbb{N}_{>0}, \lesssim, \mathcal{I}_{n}\right)$ where $\lesssim$ is the inverse of the usual order notion on $\mathbb{N}$ (namely, $m \lesssim n$ iff $m \geq n$ ) and, for every natural number $n, \mathcal{I}_{n}:=\{m \in \mathbb{N} \mid m \lesssim n\}$. If $f: \mathbb{N}_{>0} \rightarrow(0,1]$ is the function that maps $n>0$ to $\frac{1}{n}$, we have that $f: \mathbb{I}^{\mathrm{s}} \longrightarrow \overline{\mathbb{N}}$ is a morphism. Conversely, if $g:(0,1] \longrightarrow \mathbb{N}$ is the function that maps $\varepsilon$ to the floor $\left\lfloor\frac{1}{\varepsilon}\right\rfloor$ then $g: \overline{\mathbb{N}} \longrightarrow \mathbb{I}^{\mathrm{S}}$ is a morphism. We have $g \circ f=1_{\mathbb{N}_{>0}}$, but there does not exist any isomorphism between these two sets of indices $\overline{\mathbb{N}}$ and $\mathbb{I}^{\mathrm{s}}$ because they have different cardinalities.

(vii) For every $n \in \mathbb{N}$ let us fix $\varphi_{n} \in \mathcal{A}_{n} \backslash \mathcal{A}_{n+1}$. Let $f: \mathbb{N} \longrightarrow \mathcal{A}_{0}$ be the function that maps $n$ to $\varphi_{n}$. Then we have that $f: \widetilde{\mathbb{I}}^{\mathrm{e}} \longrightarrow \overline{\mathbb{N}}$ is a morphism. Conversely, if $o: \mathcal{A}_{0} \longrightarrow \overline{\mathbb{N}}$ is the function that maps $\varphi$ to $o(\varphi)$ (see (iv) in Example 2) then $o: \overline{\mathbb{N}} \longrightarrow \widetilde{\mathbb{I}}^{\mathrm{e}}$ is a morphism.

Lemma 10 Let $\mathbb{I}_{k}=\left(I_{k}, \leq_{k}, \mathcal{I}_{k}\right)$ be sets of indices for $k=1$, 2, 3. Let a $\in A \in$ $\mathcal{I}_{1}, b \in B \in \mathcal{I}_{2}$ and $c \in C \in \mathcal{I}_{3}$. Then if $f: A_{\leq a} \longrightarrow B_{\leq b}$ and $g: B_{\leq b} \longrightarrow C_{\leq c}$ are infinitesimals, also the composition $f \circ g: A_{\leq a} \longrightarrow C_{\leq c}$ is infinitesimal.

Proof By definition, for every $\alpha \in A_{\leq a}$ there exists $\delta_{2} \leq b$ such that $f\left(\varepsilon_{2}\right) \in A_{\leq \alpha}$ for every $\varepsilon_{2} \in B$ such that $\varepsilon_{2} \leq \delta_{2} \leq \bar{b}$. But $g: B_{\leq b} \longrightarrow C_{\leq c}$ infinitesimal means

$$
\forall \beta \in B_{\leq b} \forall^{\mathbb{I}_{3}} \varepsilon_{3} \in C_{\leq c}: g\left(\varepsilon_{3}\right) \in B_{\leq \beta} .
$$

We apply this property with $\beta=\delta_{2}$ to get $g\left(\varepsilon_{3}\right) \in B_{\leq \delta_{2}}$ for every $\varepsilon_{3} \in C_{\leq c}$ sufficiently small, let us say for each $\varepsilon_{3} \leq \delta_{3} \leq c$. Therefore $f\left(g\left(\varepsilon_{3}\right)\right) \in A_{\leq \alpha}$ for every $\varepsilon_{3} \in C_{\leq c}$ such that $\varepsilon_{3} \leq \delta_{3}$.

The following results motivate our definition of morphism of sets of indices. 
Lemma 11 In the assumptions of Definition 8, let $f: A_{\leq a} \longrightarrow B_{\leq b}$ be infinitesimal, and let $\mathcal{P}\left(\varepsilon_{1}\right)$ be a given property of $\varepsilon_{1} \in I_{1}$. If $\mathcal{P}\left(\varepsilon_{1}\right)$ holds $\forall^{\mathbb{I}_{1}} \varepsilon_{1} \in A_{\leq a}$ then $\mathcal{P}\left(f\left(\varepsilon_{2}\right)\right)$ holds $\forall^{\mathbb{I}_{2}} \varepsilon_{2} \in B_{\leq b}$.

Proof Let $e_{1} \in A_{\leq a}$ be such that $\mathcal{P}\left(\varepsilon_{1}\right)$ holds for all $\varepsilon_{1} \in A_{\leq e_{1}}$. Since $f: A_{\leq a} \longrightarrow$ $B_{\leq b}$ is infinitesimal, there exists $e_{2} \in B_{\leq b}$ be such that $f\left(\varepsilon_{2}\right) \in A_{\leq e_{1}}$ for all $\varepsilon_{2} \in B_{\leq e_{2}}$. Therefore $\mathcal{P}\left(f\left(\varepsilon_{2}\right)\right)$ holds for all $\varepsilon_{2} \in \bar{B}_{\leq e_{2}}$.

Theorem 12 Let $\mathbb{I}_{k}=\left(I_{k}, \leq_{k}, \mathcal{I}_{k}\right)$ be sets of indices for $k=1$, 2. Let $f: \mathbb{I}_{1} \longrightarrow \mathbb{I}_{2}$ be a morphism of sets of indices and let $\mathcal{P}\left(\varepsilon_{1}\right)$ be a given property of $\varepsilon_{1} \in I_{1}$. If $\forall^{\mathbb{I}_{1}} \varepsilon_{1} \mathcal{P}\left(\varepsilon_{1}\right)$ then $\forall^{\mathbb{I}_{2}} \varepsilon_{2} \mathcal{P}\left(f\left(\varepsilon_{2}\right)\right)$.

Proof Let $A \in \mathcal{I}_{1}$ be such that $\forall a \in A \forall^{\mathbb{I}_{1}} \varepsilon_{1} \in A_{\leq a} \mathcal{P}\left(\varepsilon_{1}\right)$ holds. Since $\emptyset \notin \mathcal{I}_{1}$, there exists $a \in A$. But $f: \mathbb{I}_{1} \longrightarrow \mathbb{I}_{2}$ is a morphism, so there exists $B \in \mathcal{I}_{2}$ such that $f: A_{\leq a} \longrightarrow B_{\leq b}$ is infinitesimal for all $b \in B$. By Lemma 11, we deduce that $\forall^{\mathbb{I}_{2}} \varepsilon_{2} \in B_{\leq b} \mathcal{P}\left(f\left(\varepsilon_{2}\right)\right)$, which is our conclusion.

Three simple consequences of Theorem 12 are presented in the following corollary.

Corollary 13 Let $\mathbb{I}_{k}=\left(I_{k}, \leq_{k}, \mathcal{I}_{k}\right)$ be sets of indices for $k=1$, 2. If $f: \mathbb{I}_{1} \longrightarrow \mathbb{I}_{2}$ is a morphism of sets of indices, then the following properties hold:

(i) If $i>\mathbb{I}_{1} j$ then $i \circ f>\mathbb{I}_{2} j \circ f$;

(ii) If $x_{\varepsilon_{1}}=O\left(y_{\varepsilon_{1}}\right)$ as $\varepsilon_{1} \in \mathbb{I}_{1}$, then $x_{f\left(\varepsilon_{2}\right)}=O\left(y_{f\left(\varepsilon_{2}\right)}\right)$ as $\varepsilon_{2} \in \mathbb{I}_{2}$;

(iii) For every net $g: I_{1} \longrightarrow \mathbb{R}$ if $l=\lim _{\mathbb{I}_{1}} g$ then $l=\lim _{\mathbb{I}_{2}} g \circ f$.

Proof Property (i) follows directly from Theorem 12 because $i>_{\mathbb{I}_{1}} j$ means $\forall^{\mathbb{I}} \varepsilon$ : $i_{\varepsilon}>j_{\varepsilon}$. To prove (ii), let $A \in \mathcal{I}_{1}$ be such that $x_{\varepsilon_{1}}=O_{A, a}\left(y_{\varepsilon_{1}}\right)$ for all $a \in A$. Therefore, there exists $H \in \mathbb{R}_{>0}$ such that $\forall^{\mathbb{I}} \varepsilon_{1} \in A_{\leq a}\left|x_{\varepsilon_{1}}\right| \leq H \cdot\left|y_{\varepsilon_{1}}\right|$. But $A \neq \emptyset$, so we can pick $a \in A$, and $f: \mathbb{I}_{1} \longrightarrow \mathbb{I}_{2}$ yields the existence of $B \in \mathcal{I}_{2}$ such that $f: A_{\leq a} \longrightarrow B_{\leq b}$ is infinitesimal for all $b \in B$. By Lemma 11 we get $\forall^{\mathbb{I}_{2}} \varepsilon_{2} \in B_{\leq b}\left|x_{f\left(\varepsilon_{2}\right)}\right| \leq H^{\cdot} \cdot\left|y_{f\left(\varepsilon_{2}\right)}\right|$, from which the conclusion follows. Using the same ideas, we can prove (iii).

Theorem 14 The class of all sets of indices together with their morphisms forms a category Ind.

Proof The only non-trivial property to prove concerns composition, namely that for every pair of arrows $\mathbb{I}_{1} \stackrel{f}{\longrightarrow} \mathbb{I}_{2}, \mathbb{I}_{2} \stackrel{g}{\longrightarrow} \mathbb{I}_{3}$, we have that $\mathbb{I}_{1} \stackrel{f \circ g}{\longrightarrow} \mathbb{I}_{3}$ is a morphism of set of indices. By our hypotheses we know that:

$$
\begin{aligned}
& \forall A \in \mathcal{I}_{1} \forall a \in A \exists B \in \mathcal{I}_{2} \forall b \in B: f: A_{\leq a} \longrightarrow B_{\leq b} \text { is infinitesimal; } \\
& \forall B \in \mathcal{I}_{2} \forall b \in B \exists C \in \mathcal{I}_{3} \forall c \in C: g: B_{\leq b} \longrightarrow C_{\leq c} \text { is infinitesimal. }
\end{aligned}
$$

For $a \in A \in \mathcal{I}_{1}$, from (3.1) we get a non empty $B \in \mathcal{I}_{2}$. Take any element $b \in B$, so that (3.2) yields the existence of $C \in \mathcal{I}_{3}$. For $c \in C$, both (3.1) and (3.2) give that $f: A_{\leq a} \longrightarrow B_{\leq b}$ and $g: B_{\leq b} \longrightarrow C_{\leq c}$ are infinitesimal, and the conclusion follows from Lemma 10 . 


\subsection{Downward directed and segmented sets of indices}

In this section, we study suitable classes of sets of indices where the notion of morphism of the category Ind simplifies.

Definition 15 Let $\mathbb{I}=(I, \leq, \mathcal{I})$ be a set of indices, then we say that

(i) II is segmented if $\forall A \in \mathcal{I} \exists a:(\emptyset, a] \subseteq A$;

(ii) II is downward directed if $(I, \leq)$ is downward directed, i.e. for every $a, b \in I$ there exists $c \in I$ such that $c \leq a, c \leq b$.

Moreover, if $\mathbb{I}$ is downward directed, we call canonical set of indices generated by $\mathbb{I}$, and we denote it by $\overline{\mathbb{I}}$, the set of indices $\overline{\mathbb{I}}=\left(I, \leq, \mathcal{S}_{I}\right)$, where

$$
\mathcal{S}_{I}:=\{(\emptyset, a] \mid a \in I\} \cup\{I\} .
$$

Since $(I, \leq)$ is downward directed, it is immediate to prove that $\overline{\mathbb{I}}$ is a set of indices.

Example 16 (i) The sets of indices $\mathbb{I}^{\mathrm{s}}$ and $\mathbb{I}^{\mathrm{E}}$ are both segmented and downward directed.

(ii) If $\mathbb{I}=\mathbb{I}^{\mathrm{s}}$ then $\overline{\mathbb{I}}=\mathbb{I}$. If $\mathbb{I}=\mathbb{I}^{\mathrm{E}}$ then $\overline{\mathbb{I}} \neq \mathbb{I}$, but we will see in Theorem 18 that they are isomorphic in the category Ind.

(iii) If $\mathbb{I}=\widetilde{\mathbb{I}}^{\mathrm{e}}$ then $\overline{\mathbb{I}} \neq \mathbb{I}$.

As mentioned above, the notion of morphism is simplified when we work with this type of sets of indices.

Theorem 17 Let $\mathbb{I}_{1}, \mathbb{I}_{2}$ be sets of indices and let $f: I_{2} \longrightarrow I_{1}$ be a map. Let us assume that $\mathbb{I}_{1}$ is segmented and $\mathbb{I}_{2}$ is downward directed. Then the following conditions are equivalent:

(i) $f: \mathbb{I}_{1} \longrightarrow \mathbb{I}_{2}$ is a morphism of sets of indices;

(ii) $\forall a \in I_{1} \exists b \in I_{2}: f((\emptyset, b]) \subseteq(\emptyset, a]$;

(iii) $\forall a \in I_{1} \forall b \in I_{2}: f:\left(I_{1}\right)_{\leq a} \longrightarrow\left(I_{2}\right)_{\leq b}$ is infinitesimal.

Proof To prove that (i) entails (ii), let $f: \mathbb{I}_{1} \longrightarrow \mathbb{I}_{2}$ be a morphism and let $a \in I_{1}$. Setting $A=I_{1}$ in the definition of morphism, we get the existence of $B \in \mathcal{I}_{2}$ such that $f: A_{\leq a} \longrightarrow B_{\leq \bar{b}}$ is infinitesimal for each $\bar{b} \in B$. Take any $\bar{b} \in B \neq \emptyset$. Setting $\alpha=a$ in the definition of infinitesimal (Definition 8), we get the existence of $b \in B_{\leq \bar{b}} \subseteq I_{2}$ such that $f\left(\varepsilon_{2}\right) \in(\emptyset, a]$ for all $\varepsilon_{2} \in(\emptyset, b]$, which is our conclusion.

To prove that (ii) entails (iii), let $a \in I_{1}, b \in I_{2}$ and let $\bar{b} \in I_{2}$ be such that $f(\emptyset, \bar{b}] \subseteq$ (Ø,a]. Let $\alpha \in(\emptyset, a]$ and let $\widetilde{b} \in B_{\leq \bar{b}}$ be such that $f(\emptyset, \widetilde{b}] \subseteq(\emptyset, \alpha]$. Since $\left(I_{2}, \leq\right)$ is downward directed, we can find $\beta \in I_{2}$ such that $\beta \leq b, \beta \leq \widetilde{b}$. By construction, $f(\emptyset, \beta] \subseteq(\emptyset, \alpha]$ and $(\emptyset, \beta] \subseteq(\emptyset, b]=\left(I_{2}\right)_{\leq b}$. Therefore $f:\left(I_{1}\right)_{\leq a} \longrightarrow\left(I_{2}\right)_{\leq b}$ is infinitesimal.

To prove that (iii) entails (i), assume that $a \in A \in \mathcal{I}_{1}$. Set $B:=I_{2}$ and take any $b \in B$. By (iii) we obtain that $f:\left(I_{1}\right)_{\leq a} \longrightarrow\left(I_{2}\right)_{\leq b}$ is infinitesimal. Therefore, for each $\alpha \leq a$ there exists $\widetilde{\beta} \leq b$ such that we have $f\left(\varepsilon_{2}\right) \leq \alpha$ for every $\varepsilon_{2} \leq \widetilde{\beta}$. small, let's say for $\varepsilon_{2} \leq \tilde{\beta} \leq b$. But $\mathbb{I}_{1}$ is segmented, so there exists $a^{\prime}$ such that $\left(\emptyset, a^{\prime}\right] \subseteq A$. 
Once again from (iii) we also have that $f:\left(I_{1}\right)_{\leq a^{\prime}} \longrightarrow\left(I_{2}\right)_{\leq b}$ is infinitesimal. Hence for some $\bar{\beta} \leq b$ we have $f\left(\varepsilon_{2}\right) \leq a^{\prime}$ for each $\varepsilon_{2} \leq \bar{\beta}$. Since $\left(I_{2}, \leq\right)$ is downward directed, we can find $\beta \in I_{2}=B$ such that $\beta \leq \tilde{\beta}$ and $\beta \leq \bar{\beta}$. Therefore, for each $\varepsilon_{2} \leq \beta$ we have both $f\left(\varepsilon_{2}\right) \leq \alpha$ and $f\left(\varepsilon_{2}\right) \in\left(\emptyset, a^{\prime}\right] \subseteq A$. This proves that $f: A_{\leq a} \longrightarrow B_{\leq b}$ is infinitesimal, which completes the proof.

Theorem 18 Every segmented downward directed set of indices $\mathbb{I}$ is isomorphic to $\overline{\mathbb{I}}$ in the category Ind.

Proof It suffices to consider the identity $1_{I}: i \in I \mapsto i \in I$, which is a morphism $1_{I} \in \operatorname{Ind}(\mathbb{I}, \overline{\mathbb{I}}) \cap \operatorname{Ind}(\overline{\mathbb{I}}, \mathbb{I})$ because of condition (ii) of Theorem 17 .

Therefore, up to isomorphism, the only segmented downward directed set of indices having $(I, \leq)$ as underlying pre-ordered set is $\overline{\mathbb{I}}$.

\section{Asymptotic gauge Colombeau type algebras}

\subsection{Asymptotic gauges}

In [12], we introduced the notion of asymptotic gauge. The idea was to use it as an asymptotic scale that generalizes the role of the polynomial family $\left(\varepsilon^{n}\right)_{\varepsilon \in(0,1], n \in \mathbb{N}}$ in classical constructions of Colombeau algebras. We recall the notations and notions from [12] that we will use in the present work. For all the proofs, we refer to [12].

Definition 19 Let $\mathbb{I}=(I, \leq, \mathcal{I})$ be a set of indices. All big-Os in this definition have to be meant as $O_{\mathcal{I}}$ in $\mathbb{I}$ (see Definition 4 ). We say that $\mathcal{B}$ is an asymptotic gauge on $\mathbb{I}$ (briefly: $\mathrm{AG}$ on $\mathbb{I}$ ) if

(i) $\mathcal{B} \subseteq \mathbb{R}^{I}$;

(ii) $\exists i \in \mathcal{B}: \lim _{\mathbb{I}} i=\infty$;

(iii) $\forall i, j \in \mathcal{B} \exists p \in \mathcal{B}: i \cdot j=O(p)$;

(iv) $\forall i \in \mathcal{B} \forall r \in \mathbb{R} \exists \sigma \in \mathcal{B}: r \cdot i=O(\sigma)$;

(v) $\forall i, j \in \mathcal{B} \exists s \in \mathcal{B}: s>\mathbb{I} 0,|i|+|j|=O(s)$.

Let $\mathcal{B}$ be an $\mathrm{AG}$ on the set of indices $\mathbb{I}=(I, \leq, \mathcal{I})$. The set of moderate nets generated by $\mathcal{B}$ is

$$
\mathbb{R}_{M}(\mathcal{B}):=\left\{x \in \mathbb{R}^{I} \mid \exists b \in \mathcal{B}: x_{\varepsilon}=O\left(b_{\varepsilon}\right)\right\}
$$

Let us observe that $\mathbb{R}_{M}(\mathcal{B})$ is an $\mathrm{AG}$, and that $\mathbb{R}_{M}\left(\mathbb{R}_{M}(\mathcal{B})\right)=\mathbb{R}_{M}(\mathcal{B})$. Every asymptotic gauge formalizes a notion of "growth condition". We can hence use an asymptotic gauge $\mathcal{B}$ to define moderate nets. We can also use the reciprocals of nets taken from another asymptotic gauge $\mathcal{Z}$ to define negligible nets. From this point of view, it is natural to introduce the following:

Definition 20 Let $\Omega \subseteq \mathbb{R}^{n}$ be an open set, let $\mathcal{B}, \mathcal{Z}$ be AGs on the same set of indices $\mathbb{I}=(I, \leq, \mathcal{I})$. The set of $\mathcal{B}$-moderate nets is

$\mathcal{E}_{M}(\mathcal{B}, \Omega):=\left\{u \in \mathcal{C}^{\infty}(\Omega)^{I}\left|\forall K \Subset \Omega \forall \alpha \in \mathbb{N}^{n} \exists b \in \mathcal{B}: \sup _{x \in K}\right| \partial^{\alpha} u_{\varepsilon}(x) \mid=O\left(b_{\varepsilon}\right)\right\}$. 
The set of $\mathcal{Z}$-negligible nets is

$$
\mathcal{N}(\mathcal{Z}, \Omega):=\left\{u \in \mathcal{C}^{\infty}(\Omega){ }^{I}\left|\forall K \Subset \Omega \forall \alpha \in \mathbb{N}^{n} \forall z \in \mathcal{Z}_{>0}: \sup _{x \in K}\right| \partial^{\alpha} u_{\varepsilon}(x) \mid=O\left(z_{\varepsilon}^{-1}\right)\right\} .
$$

In [12], we proved that if $\mathbb{R}_{M}(\mathcal{B}) \subseteq \mathbb{R}_{M}(\mathcal{Z})$ then the quotient $\mathcal{E}_{M}(\mathcal{B}, \Omega) / \mathcal{N}(\mathcal{Z}, \Omega)$ is an algebra. When this happens, we will use the following:

Definition 21 Let $\mathcal{B}, \mathcal{Z}$ be AGs on the same set of indices $\mathbb{I}=(I, \leq, \mathcal{I})$ such that $\mathbb{R}_{M}(\mathcal{B}) \subseteq \mathbb{R}_{M}(\mathcal{Z})$. The Colombeau $A G$ algebra generated by $\mathcal{B}$ and $\mathcal{Z}$ is the quotient

$$
\mathcal{G}(\mathcal{B}, \mathcal{Z}):=\mathcal{E}_{M}(\mathcal{B}, \Omega) / \mathcal{N}(\mathcal{Z}, \Omega)
$$

Moreover, we set

(i) $\left(x_{\varepsilon}\right) \sim_{\mathcal{Z}}\left(y_{\varepsilon}\right)$ if and only if $\forall z \in \mathcal{Z}_{>0}: x_{\varepsilon}-y_{\varepsilon}=O\left(z_{\varepsilon}^{-1}\right)$, where $\left(x_{\varepsilon}\right)$, $\left(y_{\varepsilon}\right) \in \mathbb{R}_{M}(\mathcal{B})$.

(ii) $\widetilde{\mathbb{R}}(\mathcal{B}, \mathcal{Z}):=\mathbb{R}_{M}(\mathcal{B}) / \sim_{\mathcal{Z}}$. We simply use the notation $\widetilde{\mathbb{R}}(\mathcal{B})$ for $\widetilde{\mathbb{R}}(\mathcal{B}, \mathcal{B})$.

We will use the notation $\mathcal{G}(\mathcal{B}, \mathcal{Z}, \Omega)$ to emphasize the dependence on the open set $\Omega$.

Example Both Egorov algebra $\mathcal{G}^{\mathrm{E}}(\Omega)$ and the algebra of non-standard smooth functions ${ }^{*} \mathcal{C}^{\infty}(\Omega)$ are Colombeau AG algebras with $\mathcal{B}=\mathcal{Z}=\mathbb{R}^{\mathbb{N}}$. In fact, in both cases $\mathcal{E}_{M}(\mathcal{B}, \Omega)=\mathcal{C}^{\infty}(\Omega)^{\mathbb{N}}$ because the AG $\mathcal{B}=\mathbb{R}^{\mathbb{N}}$ is trivial. It is also easy to see that in the former case $\left(u_{n}\right)_{n \in \mathbb{N}} \in \mathcal{N}^{\mathrm{E}}\left(\mathbb{R}^{\mathbb{N}}, \Omega\right)$ if and only if for all $K \Subset \Omega,\left.u_{n}\right|_{K}=0$ for all $n \in \mathbb{N}$ sufficiently big. In the latter $\left(u_{n}\right)_{n \in \mathbb{N}} \in \mathcal{N}^{*}\left(\mathbb{R}^{\mathbb{N}}, \Omega\right)$ if and only if for all $K \Subset \Omega$ there exists an ultrafilter set $A \in \mathcal{I}^{*}$ such that $\left.u_{n}\right|_{K}=0$ for all $n \in A$.

Morphisms between sets of indices can be used to construct asymptotic gauges, as the following theorem shows.

Theorem 22 Let $\mathcal{B}$ be an asymptotic gauge on the set of indices $\mathbb{I}_{1}$ and let $f: \mathbb{I}_{1} \longrightarrow$ $\mathbb{I}_{2}$ be a morphism. Then

$$
\mathcal{B} \circ f=\{b \circ f \mid b \in \mathcal{B}\}
$$

is an asymptotic gauge on $\mathbb{I}_{2}$.

Proof All the defining properties of an asymptotic gauge for $\mathcal{B} \circ f$ can be derived from Corollary 13. For example, let us prove that $\forall i, j \in \mathcal{B} \circ f \exists s \in \mathcal{B} \circ f: s>\mathbb{I}$ $0,|i|+|j|=O(s)$. Let $i, j \in \mathcal{B} \circ f$ and let $i=b_{1} \circ f, j=b_{2} \circ f$. Let $b_{3} \in \mathcal{B}$ be such that $\left|b_{1}\right|+\left|b_{2}\right|=O\left(b_{3}\right)$. Then by Corollary 13 we deduce that $\left|b_{1} \circ f\right|+\left|b_{2} \circ f\right|=$ $O\left(b_{3} \circ f\right)$. Setting $s=b_{3} \circ f$ we therefore have that $|i|+|j|=O(s)$. 


\section{The categories $\mathrm{Ag}_{2}$ and $\mathrm{Ag}_{1}$}

We want to prove that the Colombeau AG algebra construction of Definition 21 is functorial in the pair $(\mathcal{B}, \mathcal{Z})$ of $A G$ s. In proving this result, the following category arises naturally:

\section{Definition 23 We set}

(i) $(\mathcal{B}, \mathcal{Z}) \in \mathrm{AG}_{2}$ if $\mathcal{B}, \mathcal{Z}$ are AGs on some set of indices $\mathbb{I}$ and $\mathbb{R}_{M}(\mathcal{B}) \subseteq \mathbb{R}_{M}(\mathcal{Z})$.

(ii) Let $\left(\mathcal{B}_{1}, \mathcal{Z}_{1}\right),\left(\mathcal{B}_{2}, \mathcal{Z}_{2}\right) \in \mathrm{AG}_{2}$ be pairs of AGs on the sets of indices resp. $\mathbb{I}_{1}$, $\mathbb{I}_{2}$. We say that $f \in \operatorname{AG}_{2}\left(\left(\mathcal{B}_{1}, \mathcal{Z}_{1}\right),\left(\mathcal{B}_{2}, \mathcal{Z}_{2}\right)\right)$ is a morphism of pairs of $A G s$ if $f \in \operatorname{Ind}\left(\mathbb{I}_{1}, \mathbb{I}_{2}\right), \mathbb{R}_{M}\left(\mathcal{B}_{1} \circ f\right) \subseteq \mathbb{R}_{M}\left(\mathcal{B}_{2}\right)$ and $\mathbb{R}_{M}\left(\mathcal{Z}_{2}\right) \subseteq \mathbb{R}_{M}\left(\mathcal{Z}_{1} \circ f\right)$.

Theorem $24 \mathrm{AG}_{2}$ with set-theoretical composition and identity is a category.

Proof It is sufficient to consider the composition. Let $f \in \mathrm{AG}_{2}\left(\left(\mathcal{B}_{1}, \mathcal{Z}_{1}\right),\left(\mathcal{B}_{2}, \mathcal{Z}_{2}\right)\right)$ and $g \in \operatorname{AG}_{2}\left(\left(\mathcal{B}_{2}, \mathcal{Z}_{2}\right),\left(\mathcal{B}_{3}, \mathcal{Z}_{3}\right)\right)$. By definition, $f \in \operatorname{Ind}\left(\mathbb{I}_{1}, \mathbb{I}_{2}\right)$ and $g \in \operatorname{Ind}\left(\mathbb{I}_{2}, \mathbb{I}_{3}\right)$, therefore $f \circ g \in \mathbf{I n d}\left(\mathbb{I}_{1}, \mathbb{I}_{3}\right)$ by Theorem 14. Moreover,

$$
\mathbb{R}_{M}\left(\mathcal{B}_{1} \circ(f \circ g)\right)=\left\{b \circ f \circ g \mid b \in \mathcal{B}_{1}\right\} \subseteq\left\{b \circ g \mid b \in \mathcal{B}_{2}\right\}=\mathcal{B}_{2} \circ g,
$$

since $\mathbb{R}_{M}\left(\mathcal{B}_{1} \circ f\right) \subseteq \mathbb{R}_{M}\left(\mathcal{B}_{2}\right)$. But $\mathcal{B}_{2} \circ g \subseteq \mathbb{R}_{M}\left(\mathcal{B}_{2} \circ g\right) \subseteq \mathbb{R}_{M}\left(\mathcal{B}_{3}\right)$, from which the first part of the conclusion follows. To prove the second part of the conclusion we notice that, as $\mathbb{R}_{M}\left(\mathcal{Z}_{2}\right) \subseteq \mathbb{R}_{M}\left(\mathcal{Z}_{1} \circ f\right)$, we have that

$$
\mathbb{R}_{M}\left(\mathcal{Z}_{2} \circ g\right) \subseteq \mathbb{R}_{M}\left(\left(\mathcal{Z}_{1} \circ f\right) \circ g\right)=\mathbb{R}_{M}\left(\mathcal{Z}_{1} \circ(f \circ g)\right),
$$

and the thesis follows since $\mathbb{R}_{M}\left(\mathcal{Z}_{3}\right) \subseteq \mathbb{R}_{M}\left(\mathcal{Z}_{2} \circ g\right)$ by hypothesis.

The generalization with two AGs is a relatively new step in considering Colombeau like algebras, and its main aim is to highlight what peculiar properties are used to derive the fundamental properties (a)-(e), in particular the specific embedding property (e). It is therefore natural to consider also the following

Definition 25 We say that $\mathcal{B} \in \mathrm{AG}_{1}$ if $(\mathcal{B}, \mathcal{B}) \in \mathrm{AG}_{2}$. We set $f \in \mathrm{AG}_{1}\left(\mathcal{B}_{1}, \mathcal{B}_{2}\right)$ if $f \in \operatorname{AG}_{2}\left(\left(\mathcal{B}_{1}, \mathcal{B}_{1}\right),\left(\mathcal{B}_{2}, \mathcal{B}_{2}\right)\right)$. We call such an $f$ a morphism of $A G$ s. In this case, Definition 23 (ii) becomes $\mathbb{R}_{M}\left(\mathcal{B}_{1} \circ f\right)=\mathbb{R}_{M}\left(\mathcal{B}_{2}\right)$.

Of course $A_{G}$ is embedded into $A_{2}$ by means of $\mathcal{B} \mapsto(\mathcal{B}, \mathcal{B})$ and of the identity on arrows. By an innocuous abuse of language, we can hence say that $A_{G_{1}}$ is a subcategory of $\mathrm{AG}_{2}$.

Example 26 (i) Let $\mathbb{I}=\mathbb{I}^{\mathrm{s}}$, let $\mathcal{B}_{1}=\left\{\left(\varepsilon^{-n}\right) \mid n \in \mathbb{N}\right\}, \mathcal{B}_{2}=\left\{\left(\varepsilon^{-2 n}\right) \mid n \in \mathbb{N}\right\}$. Then $f, g: \mathbb{I} \longrightarrow \mathbb{I}$ such that $f(\varepsilon)=\varepsilon^{2}$ and $g(\varepsilon)=\sqrt{\varepsilon}$ induce morphisms $\mathcal{B}_{1} \stackrel{f}{\longrightarrow} \mathcal{B}_{2}$ and $\mathcal{B}_{2} \stackrel{g}{\longrightarrow} \mathcal{B}_{1}$. Clearly $f \circ g=1_{\mathcal{B}_{2}}$ and $g \circ f=1_{\mathcal{B}_{1}}$, therefore $\mathcal{B}_{1}$ and $\mathcal{B}_{2}$ are isomorphic.

(ii) Let $\mathbb{I}_{1}=\mathbb{I}^{\mathrm{s}}, \mathbb{I}_{2}=\overline{\mathbb{N}}$ (see Example 9 (vi)), let $\mathcal{B}_{1}=\left\{\left(\varepsilon^{-n}\right) \mid n \in \mathbb{N}\right\}, \mathcal{B}_{2}=$ $\left\{\left(n^{m}\right)_{n} \mid m \in \mathbb{N}\right\}$. Then $f: \mathbb{I}_{1} \longrightarrow \mathbb{I}_{2}$ such that $f(n)=\frac{1}{n+1}$ for every $n \in \mathbb{N}$ induces a morphism $\mathcal{B}_{1} \stackrel{f}{\longrightarrow} \mathcal{B}_{2}$ and $g: \mathbb{I}_{2} \longrightarrow \mathbb{I}_{1}$ such that $g(\varepsilon)=\left\lfloor\frac{1}{\varepsilon}\right\rfloor$ for every $\varepsilon \in(0,1]$ induces a morphism $\mathcal{B}_{2} \stackrel{g}{\longrightarrow} \mathcal{B}_{1}$. 
(iii) Let $o: \overline{\mathbb{N}} \longrightarrow \widetilde{\mathbb{I}}^{\mathrm{e}}$ be the morphism given by the maps $o$ that maps every $\varphi \in \mathcal{A}_{0}$ to the order $o(\varphi)$ of $\varphi$. Let $\mathcal{B}_{1}=\left\{\left(b_{n}^{m}\right)_{n} \mid m \in \mathbb{N}\right\}$ where $b_{n}=\frac{1}{n+1}$ for every $n \in \mathbb{N}$ and let $\mathcal{B}_{2}=\left\{\left(b_{\varphi}^{n}\right)_{\varphi} \mid n \in \mathbb{N}\right\}$, where $b_{\varphi}=\frac{1}{o(\varphi)+1}$ for every $\varphi \in \mathcal{A}_{0}$. Then $\mathcal{B}_{1} \stackrel{o}{\longrightarrow} \mathcal{B}_{2}$ is a morphism.

(iv) Set $f(\varepsilon)=\varepsilon+\varepsilon^{2} \cdot \sin \left(\frac{1}{\varepsilon}\right)$ for $\varepsilon \in(0,1]$ and $\mathcal{B}^{\mathrm{s}}:=\left\{\varepsilon^{-a} \mid a \in \mathbb{R}_{>0}\right\}$. Then $\varepsilon-\varepsilon^{2} \leq f(\varepsilon) \leq \varepsilon+\varepsilon^{2}$, and this implies $f \in \mathrm{AG}_{1}\left(\mathcal{B}^{\mathrm{s}}, \mathcal{B}^{\mathrm{s}}\right)$. Let us note that $f$ is not invertible in any neighbourhood of 0 so that it is not an isomorphism of AGs.

In [12], we defined two asymptotic gauges $\mathcal{B}_{1}, \mathcal{B}_{2}$ to be equivalent if and only if $\mathbb{R}_{M}\left(\mathcal{B}_{1}\right)=\mathbb{R}_{M}\left(\mathcal{B}_{2}\right)$. Within the present categorical framework, this definition is motivated by the following result.

Theorem 27 Let $\mathcal{B}$ be an asymptotic gauge on $\mathbb{I}$. Then $\mathcal{B}$ is isomorphic to $\mathbb{R}_{M}(\mathcal{B})$.

Proof It is sufficient to observe that, by definition, $\mathcal{B} \stackrel{1_{I}}{\longrightarrow} \mathbb{R}_{M}(\mathcal{B})$ is a morphism and, since $\mathbb{R}_{M}\left(\mathbb{R}_{M}(\mathcal{B})\right)=\mathbb{R}_{M}(\mathcal{B})$, also $\mathbb{R}_{M}(\mathcal{B}) \stackrel{1_{I}}{\longrightarrow} \mathcal{B}$ is a morphism.

In particular, it follows that for every two asymptotic gauges $\mathcal{B}_{1}, \mathcal{B}_{2}$ defined on the same set of indices $\mathbb{I}$, we have that if $\mathcal{B}_{1}$ is equivalent to $\mathcal{B}_{2}$ then they are isomorphic. Conversely, if $f \in \operatorname{AG}_{1}\left(\mathcal{B}_{1}, \mathcal{B}_{2}\right)$ is an isomorphism, then $\mathbb{R}_{M}\left(\mathcal{B}_{1}\right)=\mathbb{R}_{M}\left(\mathcal{B}_{1} \circ f \circ f^{-1}\right)=$ $\mathbb{R}_{M}\left(\mathcal{B}_{2} \circ f^{-1}\right)=\mathbb{R}_{M}\left(\mathcal{B}_{1}\right)$, and hence $\mathbb{R}_{M}\left(\mathcal{B}_{1}\right)=\mathbb{R}_{M}\left(\mathcal{B}_{2} \circ f^{-1}\right)$. Analogously, $\mathbb{R}_{M}\left(\mathcal{B}_{2}\right)=\mathbb{R}_{M}\left(\mathcal{B}_{1} \circ f\right)$. In particular, the identity $1_{I} \in \mathrm{AG}_{1}\left(\mathcal{B}_{1}, \mathcal{B}_{2}\right) \cap \operatorname{AG}_{1}\left(\mathcal{B}_{2}, \mathcal{B}_{1}\right)$ if and only if these AGs are equivalent. For example $\left\{\left(\varepsilon^{-a}\right) \mid a \in \mathbb{R}_{>0}\right\}$ and $\left\{\left(\varepsilon^{-n}\right) \mid n \in \mathbb{N}\right\}$ are equivalent. Nevertheless, it is not difficult to prove that not all isomorphic AGs on the same set of indices are equivalent. To prove this result, we need to recall (see [12, Def. 36]) that an AG $\mathcal{B}$ is called principal if there exists a generator $b \in \mathcal{B}$ such that $\mathbb{R}_{M}(\mathrm{AG}(b))=\mathbb{R}_{M}(\mathcal{B})$, where $\mathrm{AG}(b):=\left\{b^{m} \mid m \in \mathbb{N}\right\}$.

Theorem 28 For every principal $A G s \mathcal{B}_{1}, \mathcal{B}_{2}$ on $\mathbb{I}^{\mathrm{s}}$, if $\mathbb{R}_{M}\left(\mathcal{B}_{1}\right) \subsetneq \mathbb{R}_{M}\left(\mathcal{B}_{2}\right)$ then there exists a principal $A G \mathcal{B}_{3}$ such that $\mathbb{R}_{M}\left(\mathcal{B}_{1}\right) \subsetneq \mathbb{R}_{M}\left(\mathcal{B}_{3}\right) \subsetneq \mathbb{R}_{M}\left(\mathcal{B}_{2}\right)$.

Proof Let $\mathcal{B}_{1}=A G\left(b_{1}\right)$ and $\mathcal{B}_{2}=A G\left(b_{2}\right)$. Without loss of generality we can assume that $b_{1, \varepsilon}, b_{2, \varepsilon}>1$ for every $\varepsilon \in(0,1]$. Moreover, as $\mathbb{R}_{M}\left(\mathcal{B}_{1}\right) \subsetneq \mathbb{R}_{M}\left(\mathcal{B}_{2}\right)$, we have $b_{1}=O_{\mathcal{I}^{\mathrm{s}}}\left(b_{2}\right)$. So, without loss of generality, we can also assume that $b_{1, \varepsilon}<b_{2, \varepsilon}$ for every $\varepsilon \in(0,1]$. Since $\mathbb{R}_{M}\left(\mathcal{B}_{1}\right) \subsetneq \mathbb{R}_{M}\left(\mathcal{B}_{2}\right)$, we have that $b_{2} \notin \mathbb{R}_{M}\left(b_{1}\right)$, namely that

$$
\forall n \in \mathbb{N} \forall \varepsilon \in(0,1] \exists \delta<\varepsilon: n \cdot b_{1, \delta}^{n}<b_{2, \delta} .
$$

Now, we let $b_{3} \in \mathbb{R}^{(0,1]}$ be a net such that $b_{1, \varepsilon} \leq b_{3, \varepsilon} \leq b_{2, \varepsilon}$ for $\varepsilon$ small, and $\forall n \in \mathbb{N}$

$$
b_{3, \bar{\varepsilon}_{n}}= \begin{cases}b_{1, \bar{\varepsilon}_{n}} & \text { if } n \text { is odd; } \\ b_{2, \bar{\varepsilon}_{n}} & \text { if } n \text { is even, }\end{cases}
$$

where $\bar{\varepsilon}_{1}=1$ and

$$
\bar{\varepsilon}_{n} \in\left\{\varepsilon<\min \left\{\frac{1}{n}, \bar{\varepsilon}_{n-1}\right\} \mid n \cdot b_{1, \varepsilon}^{n}<b_{2, \varepsilon}\right\}
$$


for every $n \geq 2$. Since $b_{1, \varepsilon} \leq b_{3, \varepsilon} \leq b_{2, \varepsilon}$ for $\varepsilon$ small, we have that $\mathbb{R}_{M}\left(\mathcal{B}_{1}\right) \subseteq$ $\mathbb{R}_{M}\left(A G\left(b_{3}\right)\right) \subseteq \mathbb{R}_{M}\left(\mathcal{B}_{2}\right)$. Let us prove that the reverse inclusions do not hold. To prove that, let us assume, by contradiction, that $\mathbb{R}_{M}\left(A G\left(b_{3}\right)\right) \subseteq \mathbb{R}_{M}\left(\mathcal{B}_{1}\right)$. In particular, there exists $k \in \mathbb{N}$ such that $b_{3}=O\left(b_{1}^{k}\right)$, namely there exists $\varepsilon \in(0,1], H \in \mathbb{R}_{>0}$ such that $b_{3, \delta} \leq H \cdot b_{1, \delta}^{k}$ for all $\delta<\varepsilon$. Set

$$
N:=\min \left\{n \in \mathbb{N} \mid n \text { is even and } n \geq \max \left\{\lceil H\rceil,\left\lceil\frac{1}{\varepsilon}\right\rceil, k\right\}\right\} .
$$

We have

$$
N \cdot b_{1, \bar{\varepsilon}_{N}}^{N}<b_{2, \bar{\varepsilon}_{N}}=b_{3, \bar{\varepsilon}_{N}} \leq H \cdot b_{1, \bar{\varepsilon}_{N}}^{k} \leq N \cdot b_{1, \bar{\varepsilon}_{N}}^{N}
$$

which is absurd. To prove that $\mathbb{R}_{M}\left(A G\left(b_{3}\right)\right) \subsetneq \mathbb{R}_{M}\left(\mathcal{B}_{2}\right)$, we proceed in a similar way. Let us assume, by contradiction, that $\mathbb{R}_{M}\left(\mathcal{B}_{2}\right) \subseteq \mathbb{R}_{M}\left(A G\left(b_{3}\right)\right)$. Let $k \in \mathbb{N}$ be such that $b_{2}=O\left(b_{3}^{k}\right)$. Let $\varepsilon \in(0,1], H \in \mathbb{R}_{>0}$ be such that $b_{2, \delta} \leq H \cdot b_{3, \delta}^{k}$ for all $\delta<\varepsilon$. Set

$$
N:=\min \left\{n \in \mathbb{N} \mid n \text { is odd and } n \geq \max \left\{\lceil H\rceil,\left\lceil\frac{1}{\varepsilon}\right\rceil, k\right\}\right\}
$$

We have

$$
b_{2, \bar{\varepsilon}_{N}} \leq H \cdot b_{3, \bar{\varepsilon}_{N}}^{k} \leq N \cdot b_{3, \bar{\varepsilon}_{N}}^{N}=N \cdot b_{1, \bar{\varepsilon}_{N}}^{N}<b_{2, \bar{\varepsilon}_{N}}
$$

which is absurd.

Corollary 29 For every principal $A G s \mathcal{B}_{1}, \mathcal{B}_{2}$ on $\mathbb{I}^{\mathrm{s}}$, if $\mathbb{R}_{M}\left(\mathcal{B}_{1}\right) \subsetneq \mathbb{R}_{M}\left(\mathcal{B}_{2}\right)$ then there exists an infinite sequence $\left\langle\mathcal{A}_{i} \mid i \in \mathbb{Z}\right\rangle$ of principal AGs on $\mathbb{I}^{\mathrm{s}}$ such that

$$
\mathbb{R}_{M}\left(\mathcal{B}_{1}\right) \subsetneq \cdots \subsetneq \mathbb{R}_{M}\left(\mathcal{A}_{-1}\right) \subsetneq \mathbb{R}_{M}\left(\mathcal{A}_{0}\right) \subsetneq \mathbb{R}_{M}\left(\mathcal{A}_{1}\right) \subsetneq \cdots \subsetneq \mathbb{R}_{M}\left(\mathcal{B}_{2}\right)
$$

Proof This is an immediate consequence of Theorem 28.

In particular, if we let $\mathcal{B}_{\text {pol }}:=\left\{\left(\varepsilon^{-n}\right) \mid n \in \mathbb{N}\right\}$ and $\mathcal{B}_{\exp }:=\left\{\left(e^{n / \varepsilon}\right) \mid n \in \mathbb{N}\right\}$, by Corollary 29 we have that there are infinitely many principal non equivalent AGs between $\mathcal{B}_{\text {pol }}$ and $\mathcal{B}_{\text {exp. }}$. However, as we will show in Sect. 7, $\mathcal{B}_{\text {pol }}:=\left\{\left(\varepsilon^{-n}\right) \mid n \in \mathbb{N}\right\}$ and $\mathcal{B}_{\exp }:=\left\{\left(e^{n / \varepsilon}\right) \mid n \in \mathbb{N}\right\}$ are isomorphic, and this shows that not all isomorphic AGs are equivalent.

In [12], we proved that $\mathbb{R}_{M}(\mathcal{B})$ is the minimal (with respect to inclusion) asymptotically closed solid ring containing the AG $\mathcal{B}$. Therefore, we deduce that, modulo isomorphism, all the objects in a skeleton subcategory of $A_{G_{1}}$ are asymptotically closed solid rings.

In [12], we introduced the notion of "exponential of an AG", which was crucial to study linear ODE with generalized constant coefficients. We recall its definition. 
Definition 30 Let $\mathcal{B}$ be an $A G$, and let $\mu: \mathbb{R} \longrightarrow \mathbb{R}_{\geq 0}$ be a non decreasing function such that

$$
\begin{gathered}
\lim _{x \rightarrow+\infty} \mu(x)=+\infty ; \\
\forall b \in \mathcal{B} \exists c \in \mathcal{B}: \mu\left(b_{\varepsilon}\right)^{2}<\mathbb{I} \mu\left(c_{\varepsilon}\right) .
\end{gathered}
$$

We set $\mu(\mathcal{B}):=\left\{\left(\mu\left(H \cdot b_{\varepsilon}\right)\right)_{\varepsilon} \mid H \in \mathbb{R}_{>0}, b \in \mathcal{B}\right\}$. In particular,

$$
e^{\mathcal{B}}:=\left\{e^{H \cdot b} \mid H \in \mathbb{R}_{>0}, b \in \mathcal{B}\right\}
$$

is called the exponential of $\mathcal{B}$.

The following results will be needed to prove Theorem 35 .

Lemma 31 In the hypotheses of Definition 30, we have that $\mu(\mathcal{B})$ is an $A G$.

Proof Definition 19 (ii) and [12, Lemma 18] imply the existence of $i \in \mathcal{B}$ such that $\lim _{\mathbb{I}} i=+\infty$. Therefore, our assumptions yield $\lim _{\varepsilon \in \mathbb{I}} \mu\left(i_{\varepsilon}\right)=+\infty$, which proves Definition 19 (ii) for $\mu(\mathcal{B})$. The asymptotic closure with respect to the sum of absolute values follows from monotonicity of $\mu$ and the inequality $\mu\left(H \cdot i_{\varepsilon}\right)+\mu\left(K \cdot j_{\varepsilon}\right) \leq$ $2 \cdot \mu\left(H \cdot\left|i_{\varepsilon}\right|+K \cdot\left|j_{\varepsilon}\right|\right)$. The asymptotic closure with respect to product follows from the inequality

$$
\mu\left(H \cdot i_{\varepsilon}\right) \cdot \mu\left(K \cdot j_{\varepsilon}\right) \leq \mu\left(H \cdot\left|i_{\varepsilon}\right|+K \cdot\left|j_{\varepsilon}\right|\right)^{2}
$$

and from assumption (5.1).

Lemma 32 Let $\mathbb{I}=(I, \leq, \mathcal{I})$ be a set of indices, and let $x, y, z \in \mathbb{R}^{I}$. Let $\mu$ : $\mathbb{R} \longrightarrow \mathbb{R}_{\geq 0}$ be a non decreasing function. Then $x_{\varepsilon}=O\left[\mu\left(y_{\varepsilon}\right)\right]$ and $y<\mathbb{I} z$ imply $x_{\varepsilon}=O\left[\mu\left(z_{\varepsilon}\right)\right]$.

Proof From the assumptions we get

$$
\begin{aligned}
& \exists A \in \mathcal{I} \forall a \in A: x_{\varepsilon}=O_{a A}\left[\mu\left(y_{\varepsilon}\right)\right] \\
& \exists B \in \mathcal{I} \forall b \in B \exists \varepsilon_{0} \leq b \forall \varepsilon \in B_{\leq \varepsilon_{0}}: y_{\varepsilon}<z_{\varepsilon} .
\end{aligned}
$$

Definition 1 (iii) implies the existence of $D \in \mathcal{I}$ such that $D \subseteq A \cap B$. For $d \in D$, [13, Thm. $2.8(\mathrm{x})]$ yields $x_{\varepsilon}=O_{d D}\left[\mu\left(y_{\varepsilon}\right)\right]$, and therefore, for suitable $H \in \mathbb{R}_{>0}$ and $\varepsilon_{0} \leq d, \varepsilon_{1} \leq d$, we can write $\left|x_{\varepsilon}\right| \leq H\left|\mu\left(y_{\varepsilon}\right)\right|=H \mu\left(y_{\varepsilon}\right)$ for each $\varepsilon \in D_{\leq \varepsilon_{1}}$ and $y_{\varepsilon}<z_{\varepsilon}$ for each $\varepsilon \in D_{\leq \varepsilon_{0}}$. Since $(\emptyset, d]=I_{\leq d}$ is directed, we can find $\bar{\varepsilon} \leq d, \varepsilon_{0}$, $\varepsilon_{1}$. Therefore, for each $\varepsilon \leq \bar{\varepsilon}$ we have $\left|x_{\varepsilon}\right| \leq H \mu\left(y_{\varepsilon}\right) \leq H \mu\left(z_{\varepsilon}\right)$ because $\mu$ is non decreasing. This proves our conclusion.

Corollary 33 Let $\mathcal{B}_{1}, \mathcal{B}_{2}$ be AGs on the same set of indices $\mathbb{I}$, and let $\mu: \mathbb{R} \longrightarrow$ $\mathbb{R}_{\geq 0}$ verify the assumptions of Definition 30. Then $\mathbb{R}_{M}\left(\mathcal{B}_{1}\right) \subseteq \mathbb{R}_{M}\left(\mathcal{B}_{2}\right)$ implies $\mathbb{R}_{M}\left(\mu\left(\mathcal{B}_{1}\right)\right) \subseteq \mathbb{R}_{M}\left(\mu\left(\mathcal{B}_{2}\right)\right)$. 
Proof Let $\left(y_{\varepsilon}^{\prime}\right)=\left(\mu\left(H \cdot b_{\varepsilon}^{\prime}\right)\right) \in \mu\left(\mathcal{B}_{1}\right)$, with $b^{\prime} \in \mathcal{B}_{1}$, and let $b^{\prime \prime} \in \mathcal{B}_{2}$ be such that $b^{\prime}<\mathbb{I} b^{\prime \prime}$. Then we have that $\left(y_{\varepsilon}^{\prime}\right)<\mathbb{I}\left(\mu\left(H \cdot b_{\varepsilon}^{\prime \prime}\right)\right) \in \mu\left(\mathcal{B}_{2}\right)$ since $\mu$ is monotone.

Definition 34 Let $\mathrm{AG}_{\leq}$be the subcategory of $\mathrm{AG}_{1}$ having the same objects of $\mathrm{AG}_{1}$, and arrows such that $f \in \operatorname{AG}_{\leq}\left(\mathcal{B}_{1}, \mathcal{B}_{2}\right)$ if $f \in \operatorname{IND}\left(\mathbb{I}_{1}, \mathbb{I}_{2}\right)$ and $\mathbb{R}_{M}\left(\mathcal{B}_{1} \circ f\right)=\mathbb{R}_{M}\left(\mathcal{B}_{2}\right)$. Let $\mu$ verifies the assumptions of Definition 30, and let $E_{\mu}: \mathrm{AG}_{\leq} \longrightarrow \mathrm{AG}_{\leq}$be defined on objects and maps of $\mathrm{AG}_{\leq}$as follows:

(i) $E(\mathcal{B}):=\mu(\mathcal{B})$ for each $\mathcal{B} \in \mathrm{AG}_{\leq}$;

(ii) $E(f):=f$ for each $f \in \operatorname{AG}_{\leq}\left(\mathcal{B}_{1}, \mathcal{B}_{2}\right)$.

Theorem $35 \mathrm{AG}_{\leq}$is a subcategory of $\mathrm{AG}_{1}$. If $\mu$ verifies the assumptions of Definition 30, then $E_{\mu}: \mathrm{AG}_{\leq} \longrightarrow \mathrm{AG}_{\leq}$is a functor.

Proof To prove the first part, assume that $\mathbb{R}_{M}\left(\mathcal{B}_{1} \circ f\right)=\mathbb{R}_{M}\left(\mathcal{B}_{2}\right)$ and $\mathbb{R}_{M}\left(\mathcal{B}_{2} \circ g\right)=$ $\mathbb{R}_{M}\left(\mathcal{B}_{3}\right)$. Then if $b_{1} \circ f<\mathbb{I}_{2} b_{2}$ and $b_{2} \circ g<\mathbb{I}_{3} b_{3}$, for $b_{i} \in \mathcal{B}_{i}$, then $b_{1} \circ f \circ g<\mathbb{I}_{3}$ $b_{2} \circ g<\mathbb{I}_{3} b_{3}$ by Corollary 13. If $b_{3}<\mathbb{I}_{3} b_{2} \circ g$ and $b_{2}<\mathbb{I}_{2} b_{1} \circ f$ then $b_{3}<\mathbb{I}_{3} b_{2} \circ g<\mathbb{I}_{3}$ $b_{1} \circ f \circ g$ once again by Corollary 13 . This implies that $\mathbb{R}_{M}\left(\mathcal{B}_{1} \circ(f \circ g)\right)=\mathbb{R}_{M}\left(\mathcal{B}_{3}\right)$, hence $f \circ g \in \mathrm{AG}_{\leq}\left(\mathcal{B}_{1}, \mathcal{B}_{3}\right)$. This and Corollary 33 show that $\mathrm{AG}_{\leq}$is a category. $\mathrm{By}$ Corollary 33 , we also have that $A_{\leq}$is a subcategory of $A_{1}$. To show the second part, since $E_{\mu}$ is the identity on arrows, it suffices to observe that $\mathcal{B}$ and $\mu(\mathcal{B})$ have the same set of indices for every $\mathrm{AG} \mathcal{B}$, and that $\mu\left(\mathcal{B}_{1}\right) \circ f=\mu\left(\mathcal{B}_{1} \circ f\right)$. Thus it follows by Corollary 33 that $E_{\mu}\left(\mathcal{B}_{1}\right)=\mu\left(\mathcal{B}_{1}\right) \stackrel{f}{\longrightarrow} \mu\left(\mathcal{B}_{2}\right)=E_{\mu}\left(\mathcal{B}_{2}\right)$ is an arrow in $\mathrm{AG}_{\leq}$for every arrow $\mathcal{B}_{1} \stackrel{f}{\longrightarrow} \mathcal{B}_{2}$ in $\mathrm{AG}_{\leq}$.

\section{Functoriality of Colombeau AG-algebras}

In this section, we want to prove that the $\operatorname{map}(\mathcal{B}, \mathcal{Z}, \Omega) \mapsto \mathcal{G}(\mathcal{B}, \mathcal{Z}, \Omega)$ is a functor. Clearly, $(\mathcal{B}, \mathcal{Z}) \in \mathrm{AG}_{2}$, so we need to introduce a category having open sets like $\Omega$ as objects:

Definition 36 We denote by $\mathcal{O} \mathbb{R}^{\infty}$ the category having as objects $\left\{\Omega \subseteq \mathbb{R}^{n} \mid n \in\right.$ $\mathbb{N}, \Omega$ open $\}$ and as morphisms $\mathcal{O} \mathbb{R}^{\infty}(U, V):=\mathcal{C}^{\infty}(U, V)$.

Therefore, we can now prove the following:

Theorem $37 \mathcal{G}: \mathrm{AG}_{2} \times\left(\mathcal{O R}^{\infty}\right)^{o p} \longrightarrow \mathrm{ALG}_{\mathbb{R}}$ is a functor, where $\mathrm{ALG}_{\mathbb{R}}$ is the category of commutative algebras over $\mathbb{R}$.

Proof Let $i \in \mathrm{AG}_{2}\left(\left(\mathcal{B}_{1}, \mathcal{Z}_{1}\right),\left(\mathcal{B}_{2}, \mathcal{Z}_{2}\right)\right)$ be a morphism of pairs of AGs and $h \in$ $\mathcal{C}^{\infty}\left(\Omega_{2}, \Omega_{1}\right), \Omega_{j}$ being an open set in $\mathbb{R}^{n_{j}}$. The natural definition of $\mathcal{G}(i, h)$ to get that $\mathcal{G}(i, h): \mathcal{G}\left(\mathcal{B}_{1}, \mathcal{Z}_{1}, \Omega_{1}\right) \longrightarrow \mathcal{G}\left(\mathcal{B}_{2}, \mathcal{Z}_{2}, \Omega_{2}\right)$ is a morphism of algebras is

$$
\mathcal{G}(i, h):\left[u_{\varepsilon_{1}}\right] \mapsto\left[u_{i_{\varepsilon_{2}}} \circ h\right] .
$$

To prove that this definition is correct, we assume $K_{2} \Subset \Omega_{2}$ and $\alpha \in \mathbb{N}^{n_{2}}$. Since $\left(u_{\varepsilon_{1}}\right) \in \mathcal{E}_{M}\left(\mathcal{B}_{1}, \Omega_{1}\right)$ and $h\left(K_{2}\right)=: K_{1} \Subset \Omega_{1}$, we obtain

$$
\exists b^{\prime} \in \mathcal{B}_{1}: \sup _{x \in K_{1}}\left|\partial^{\alpha} u_{\varepsilon_{1}}(x)\right|=O\left(b_{\varepsilon_{1}}^{\prime}\right) \text { as } \varepsilon_{1} \in \mathbb{I}_{1} .
$$


But $b^{\prime} \in \mathcal{B}_{1} \subseteq \mathbb{R}_{M}\left(\mathcal{B}_{1}\right)$, so $b^{\prime} \circ i \in \mathbb{R}_{M}\left(\mathcal{B}_{1} \circ i\right) \subseteq \mathbb{R}_{M}\left(\mathcal{B}_{2}\right)$. We can hence write $b_{i_{\varepsilon_{2}}}^{\prime}=O\left(b_{\varepsilon_{2}}^{\prime \prime}\right)$ as $\varepsilon_{2} \in \mathbb{I}_{2}$ for a suitable $b^{\prime \prime} \in \mathcal{B}_{2}$. This, Corollary 13 (ii) and (6.1) yield

$$
\sup _{x \in K_{2}}\left|\partial^{\alpha}\left(u_{i_{\varepsilon_{2}}} \circ h\right)(x)\right|=O\left(b_{i_{\varepsilon_{2}}}^{\prime}\right)=O\left(b_{\varepsilon_{2}}^{\prime \prime}\right) \text { as } \varepsilon_{2} \in \mathbb{I}_{2} .
$$

This shows that $\left[u_{i_{\varepsilon_{2}}} \circ h\right] \in \mathcal{E}_{M}\left(\mathcal{B}_{2}, \Omega_{2}\right)$. Now assume that $\left(u_{\varepsilon_{1}}\right)-\left(v_{\varepsilon_{1}}\right) \in \mathcal{N}\left(\mathcal{Z}_{1}, \Omega_{1}\right)$, $K_{2}, \alpha$ as above, and $z \in\left(\mathcal{Z}_{2}\right)_{>0}$. Since $\mathbb{R}_{M}\left(\mathcal{Z}_{2}\right) \subseteq \mathbb{R}_{M}\left(\mathcal{Z}_{1} \circ i\right)$, we can write $z_{\varepsilon_{2}}=$ $O\left(\zeta_{i_{\varepsilon_{2}}}\right)$ as $\varepsilon_{2} \in \mathbb{I}_{2}$ for a suitable $\zeta \in\left(\mathcal{Z}_{1}\right)_{>0}$, and hence $\zeta_{i_{\varepsilon_{2}}}^{-1}=O\left(z_{\varepsilon_{2}}^{-1}\right)$. We thus obtain

$$
\sup _{x \in K_{1}}\left|\partial^{\alpha} u_{\varepsilon_{1}}(x)-\partial^{\alpha} v_{\varepsilon_{1}}(x)\right|=O\left(\zeta_{\varepsilon_{1}}^{-1}\right) \text { as } \varepsilon_{1} \in \mathbb{I}_{1} \text {, }
$$

where $h\left(K_{2}\right)=: K_{1}$. From this and Corollary 13 (ii) we obtain the conclusion

$$
\sup _{x \in K_{2}}\left|\partial^{\alpha}\left(u_{i_{\varepsilon_{2}}} \circ h\right)(x)-\partial^{\alpha}\left(v_{i_{\varepsilon_{2}}} \circ h\right)(x)\right|=O\left(z_{\varepsilon_{2}}^{-1}\right) \text { as } \varepsilon_{2} \in \mathbb{I}_{2} .
$$

The proof that $\mathcal{G}(i, h)$ is a morphism of $\mathbb{R}$-algebras follows immediately from the pointwise definitions of the algebra operations. The functorial properties of $\mathcal{G}$ follow directly from the definition of $\mathcal{G}(i, h)$ and the fact that in the domain $\mathrm{AG}_{2} \times\left(\mathcal{O} \mathbb{R}^{\infty}\right)^{\mathrm{op}}$ composition and identity are the corresponding set-theoretical operations.

Now, let $n \in \mathbb{N}$ be fixed. Let $\mathcal{T} \mathbb{R}^{n}$ be the subcategory of $\mathcal{O} \mathbb{R}^{\infty}$ having as objects the open subsets of $\mathbb{R}^{n}$ and, as morphisms, the inclusions. From Theorem 37 we get that $\mathcal{G}(\mathcal{B}, \mathcal{Z},-):\left(\mathcal{T} \mathbb{R}^{n}\right)^{\mathrm{op}} \longrightarrow \mathrm{ALG}_{\mathbb{R}}$ is a functor, i.e. it is a presheaf. Trivially generalizing [5], it is also possible to prove that $\mathcal{G}(\mathcal{B}, \mathcal{Z},-)$ is a sheaf of differential algebras. In particular, the following diagram commutes

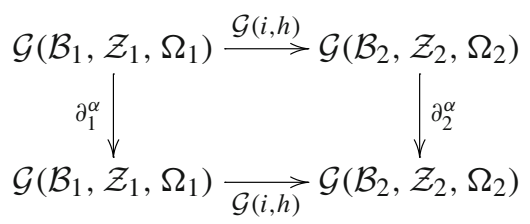

for each multi-index $\alpha \in \mathbb{N}^{n}$ and each inclusion $h \in \mathcal{T} \mathbb{R}^{n}\left(\Omega_{2}, \Omega_{1}\right)$. Clearly, in (6.2), $\partial_{k}^{\alpha}:\left[u_{\varepsilon}\right] \in \mathcal{G}\left(\mathcal{B}_{k}, \mathcal{Z}_{k}, \Omega_{k}\right) \mapsto\left[\partial^{\alpha} u_{\varepsilon}\right] \in \mathcal{G}\left(\mathcal{B}_{k}, \mathcal{Z}_{k}, \Omega_{k}\right)$. Let us note that, in general, the diagram (6.2) doesn't commute if $h$ is an arbitrary smooth function. For this reason, when we want to deal with differential algebras, we will always consider $\mathcal{T} \mathbb{R}^{n}$ instead of the category $\mathcal{O} \mathbb{R}^{\infty}$.

As a consequence of Theorem 37, we also have that essentially all the constructions of Colombeau-like algebras are functorial. For example, we can consider the set of indices $\mathbb{I}^{\mathrm{s}}$ of the special algebra, the AG $\mathcal{B}^{\mathrm{s}}:=\left\{\left(\varepsilon^{-a}\right) \mid a \in \mathbb{R}_{>0}\right\}$, and the full subcategory $\mathrm{AG}_{\mathbb{I}^{\mathrm{s}}}$ of $\mathrm{AG}_{1}$ of all the $\mathrm{AGs}$ on $\mathbb{I}^{\mathrm{s}}$. Clearly, $\mathcal{G}^{\mathrm{s}}(\mathcal{B}, \Omega):=\mathcal{G}(\mathcal{B}, \mathcal{B}, \Omega)$ is a functor $\mathcal{G}^{\mathrm{s}}: \mathrm{AG}_{\mathbb{I}^{\mathrm{s}}} \times\left(\mathcal{O} \mathbb{R}^{\infty}\right)^{\text {op }} \longrightarrow \mathrm{ALG}_{\mathbb{R}}$ which corresponds to the usual sheaf via 
the restriction $\mathcal{G}^{\mathrm{s}}\left(\mathcal{B}^{\mathrm{s}}, \Omega\right)$ only for $\Omega \in \mathcal{T} \mathbb{R}^{n}$. Analogously, we can consider $\hat{\mathcal{G}}, \mathcal{G}^{\mathrm{e}}, \mathcal{G}^{\mathrm{d}}$, $\mathcal{G}^{2}, \mathcal{G}^{\mathrm{E}}$ and ${ }^{*} \mathcal{C}^{\infty}(\Omega)$.

We also finally note that if we consider an inclusion $h \in \mathcal{T} \mathbb{R}^{n}\left(\Omega_{2}, \Omega_{1}\right)$ and a morphism of pairs of AGs $i \in \mathrm{AG}_{2}\left(\left(\mathcal{B}_{1}, \mathcal{Z}_{1}\right),\left(\mathcal{B}_{2}, \mathcal{Z}_{2}\right)\right)$, then $\mathcal{G}(i, h): \mathcal{G}\left(\mathcal{B}_{1}, \mathcal{Z}_{1}, \Omega_{1}\right) \longrightarrow$ $\mathcal{G}\left(\mathcal{B}_{2}, \mathcal{Z}_{2}, \Omega_{2}\right)$ preserves all polynomial and differential operations. Of course, it also takes generalized functions in the domain $\mathcal{G}\left(\mathcal{B}_{1}, \mathcal{Z}_{1}, \Omega_{1}\right)$ into generalized functions in the codomain $\mathcal{G}\left(\mathcal{B}_{2}, \mathcal{Z}_{2}, \Omega_{2}\right)$. We can therefore state that $\mathcal{G}(i, h)$ permits to relate differential problems framed in $\mathcal{G}\left(\mathcal{B}_{1}, \mathcal{Z}_{1}, \Omega_{1}\right)$ to those framed in $\mathcal{G}\left(\mathcal{B}_{2}, \mathcal{Z}_{2}, \Omega_{2}\right)$; see also the next Theorem 38 .

\section{An unexpected isomorphism}

If we set $\mathcal{B}_{\text {pol }}:=\left\{\left(\varepsilon^{-n}\right) \mid n \in \mathbb{N}\right\}$ and $\mathcal{B}_{\exp }:=\left\{\left(e^{n / \varepsilon}\right) \mid n \in \mathbb{N}\right\}$, it is well known (see $[5,12])$ that an ODE like

$$
\left\{\begin{array}{l}
x^{\prime}(t)-\left[\varepsilon^{-1}\right] \cdot x(t)=0 \\
x(0)=1
\end{array}\right.
$$

has no solutions in the algebra $\mathcal{G}\left(\mathcal{B}_{\text {pol }}, \Omega\right)=\mathcal{G}^{\mathrm{s}}(\Omega)$, but it has a (unique) solution $x(t)=\left[e^{\frac{1}{\varepsilon} t}\right], t \in \widetilde{\mathbb{R}}_{c}\left(\mathcal{B}_{\text {exp }}\right)$, in $\mathcal{G}\left(\mathcal{B}_{\text {exp }}, \mathbb{R}\right)$. On the other hand, if we set $\lambda(\varepsilon):=-\frac{1}{\log \varepsilon}$, for $\varepsilon \in(0,1)$, and $\lambda(1):=1$, then we have $\lim _{\varepsilon \rightarrow 0^{+}} \lambda(\varepsilon)=0^{+}$and hence, by Example 9 (ii), $\lambda \in \operatorname{IND}\left(\mathbb{I}^{\mathrm{s}}, \mathbb{I}^{\mathrm{s}}\right)$ is a morphism of set of indices. Moreover, $\left(\left(e^{n / \varepsilon}\right) \circ\right.$ $\lambda)(\varepsilon)=\varepsilon^{-n}$ and hence $\mathbb{R}_{M}\left(\mathcal{B}_{\exp } \circ \lambda\right)=\mathbb{R}_{M}\left(\mathcal{B}_{\text {pol }}\right)$. Therefore, $\lambda \in \mathrm{AG}_{1}\left(\mathcal{B}_{\text {exp }}, \mathcal{B}_{\text {pol }}\right)$ is a morphism of AGs. Analogously, if we set $\eta(\varepsilon):=e^{-\frac{1}{\varepsilon}}$, for $\varepsilon \in(0,1)$, and $\eta(1):=1$, then we have $\lambda=\eta^{-1}$ and $\eta \in \mathrm{AG}_{1}\left(\mathcal{B}_{\text {pol }}, \mathcal{B}_{\text {exp }}\right)$. Therefore $\mathcal{B}_{\text {pol }} \simeq \mathcal{B}_{\text {exp }}$ as AGs. Theorem 37 thus yields

$$
\begin{aligned}
\mathcal{G}\left(\mathcal{B}_{\mathrm{pol}}, \Omega\right) & \simeq \mathcal{G}\left(\mathcal{B}_{\mathrm{exp}}, \Omega\right) \\
\widetilde{\mathbb{R}}\left(\mathcal{B}_{\mathrm{pol}}\right) & \simeq \widetilde{\mathbb{R}}\left(\mathcal{B}_{\mathrm{exp}}\right)
\end{aligned}
$$

This does not imply that the algebra $\mathcal{G}\left(\mathcal{B}_{\text {exp }}, \mathbb{R}\right)$ is useless, because we still have the fact that the Cauchy problem (7.1) has no solution in $\mathcal{G}\left(\mathcal{B}_{\text {pol }}, \mathbb{R}\right)$. Nonetheless, we can say that the isomorphism $\lambda \in \mathrm{AG}_{1}\left(\mathcal{B}_{\text {exp }}, \mathcal{B}_{\text {pol }}\right)$ transforms (7.1) into

$$
\left\{\begin{array}{l}
x^{\prime}(t)-[-\log \varepsilon] \cdot x(t)=0 \\
x(0)=1
\end{array}\right.
$$

Therefore, (7.3) has solution in $\mathcal{G}\left(\mathcal{B}_{\text {pol }}, \mathbb{R}\right)$ if and only if (7.1) has solution in $\mathcal{G}\left(\mathcal{B}_{\text {exp }}, \mathbb{R}\right)$. The algebra $\mathcal{G}\left(\mathcal{B}_{\text {exp }}, \mathbb{R}\right)$ is the classical example introduced in literature (see e.g. $[6,8]$ ) as a completely different space with respect to the classical Colombeau algebra $\mathcal{G}\left(\mathcal{B}_{\text {pol }}, \mathbb{R}\right)$. In fact, the space $\mathcal{G}\left(\mathcal{B}_{\text {exp }}, \mathbb{R}\right)$ is usually thought of as one where we can solve differential problems which are unsolvable in $\mathcal{G}\left(\mathcal{B}_{\text {pol }}, \mathbb{R}\right)$. Clearly this is still true, but here we have showed that these two algebras are really isomorphic and 
that the solution of (7.1) is equivalent to the solution of the isomorphic problem (7.3). For these reasons, we are saying that this isomorphism is unexpected.

This example is generalized in the following theorem, where we talk, essentially for the sake of simplicity, of ODE.

Theorem 38 Let $\mathbb{I} \in \operatorname{IND}$ be a set of indices and let $b, c \in \mathbb{R}^{I}$ be infinite nets, i.e. such that $\lim _{\varepsilon \in \mathbb{I}} b_{\varepsilon}=\lim _{\varepsilon \in \mathbb{I}} c_{\varepsilon}=+\infty$. Set

$$
A G(b):=\left\{\left(b_{\varepsilon}^{n}\right) \mid n \in \mathbb{N}\right\}
$$

for the AG generated by $b$ (and analogously for $c$ ). Assume that $\eta, \lambda \in \operatorname{IND}(\mathbb{I}, \mathbb{I})$ are morphisms of $\mathbb{I}$ such that $\eta=\lambda^{-1}$ :

$$
b_{\eta(\varepsilon)}=O_{\mathcal{I}}\left(c_{\varepsilon}\right), c_{\lambda(\varepsilon)}=O_{\mathcal{I}}\left(b_{\varepsilon}\right) \text { as } \varepsilon \in \mathbb{I} .
$$

Then

(i) $A G(b) \simeq A G(c)$ as $A G s$;

(ii) $\mathcal{G}(A G(b), \Omega) \simeq \mathcal{G}(A G(c), \Omega)$ and $\widetilde{\mathbb{R}}(A G(b)) \simeq \widetilde{\mathbb{R}}(A G(c))$ in the category ALG $\mathbb{R}_{\mathbb{R}}$;

(iii) let $F=\left[F_{\varepsilon}\right] \in \mathcal{G}\left(A G(b), \mathbb{R}^{n} \times \mathbb{R}\right), \bar{x}=\left[\bar{x}_{\varepsilon}\right] \in \widetilde{\mathbb{R}}^{n}, \bar{t}=\left[\bar{t}_{\varepsilon}\right] \in \widetilde{\mathbb{R}}$. Then the Cauchy problem

$$
\left\{\begin{array}{l}
x^{\prime}(t)=F(x(t), t) \\
x(\bar{t})=\bar{x}
\end{array}\right.
$$

has a solution $x \in \mathcal{G}\left(A G(b),\left(t_{1}, t_{2}\right)\right)$ if and only if the Cauchy problem

$$
\left\{\begin{array}{l}
y^{\prime}(t)=\left[F_{\lambda(\varepsilon)}\right](y(t), t) \\
y\left(\left[\bar{t}_{\lambda(\varepsilon)}\right]\right)=\left[\bar{x}_{\lambda(\varepsilon)}\right]
\end{array}\right.
$$

has a solution $y \in \mathcal{G}\left(A G(c),\left(t_{1}, t_{2}\right)\right)$.

Proof Assumption (7.4) yields $\left(b^{n} \circ \eta\right)(\varepsilon)=b_{\eta(\varepsilon)}^{n}=O_{\mathcal{I}}\left(c_{\varepsilon}^{n}\right)$ so $\mathbb{R}_{M}(\mathrm{AG}(b) \circ \eta)=$ $\mathbb{R}_{M}(\mathrm{AG}(c))$. Analogously, we have $\mathbb{R}_{M}(\mathrm{AG}(c) \circ \lambda)=\mathbb{R}_{M}(\mathrm{AG}(b))$. This shows that $\eta$ and $\lambda$ are morphisms of AGs, and hence it proves (i). Property (ii) follows from the functorial property of $\mathcal{G}(-, \Omega): \mathrm{AG}_{1} \longrightarrow \mathrm{ALG}_{\mathbb{R}}$. To show (iii), let $x=\left[x_{\varepsilon}\right] \in$ $\mathcal{G}\left(\mathrm{AG}(b),\left(t_{1}, t_{2}\right)\right)$ be a solution of $(7.5)$ and set $y:=\mathcal{G}\left(\lambda, 1_{\left(t_{1}, t_{2}\right)}\right)(x)=\left[x_{\lambda(\varepsilon)}\right]$. Therefore, Theorem 37 and (6.2) yield the conclusion.

For instance, if $b, c:(0,1] \longrightarrow(0,1]$ are homeomorphisms such that $\lim _{\varepsilon \rightarrow 0^{+}} b_{\varepsilon}=$ $0=\lim _{\varepsilon \rightarrow 0^{+}} c_{\varepsilon}$, then $\eta:=c \circ b^{-1}$ and $\lambda:=\eta^{-1}$ verify the assumptions of this theorem.

In this categorical framework, it is unnatural to expect that isomorphisms as in (ii) hold for every pair of generators $b, c$. It is simple to see that this is the case if $b, c$ are generators defined, respectively, on two sets of indices $\mathbb{I}_{1}=\left(I_{1}, \leq_{1}, \mathcal{I}_{1}\right), \mathbb{I}_{2}=$ $\left(I_{2}, \leq_{2}, \mathcal{I}_{2}\right)$ such that $I_{1}$ and $I_{2}$ have different cardinalities, as in this case there can not 
be a bijection between $I_{1}$ and $I_{2}$. A more interesting question is if there exist principal AGs on the same set of indices that are not isomorphic. This is actually the case, as we will show after Definition 39, where we make precise the definition of the category of principal AGs.

We finally note that an isomorphism similar to (ii) has been proved by $[21,22,24]$ in the context of NSA. However, note that in $[21,22,24]$ the non-constructive isomorphism strongly depends on the condition that the cardinality of the field of generalized numbers equals the successor of $\operatorname{card}(\mathbb{R})$. On the contrary, here we have a constructive, but less general, isomorphism strictly depending on the notion of morphism of AGs and the functorial properties of Colombeau AG-algebras.

\section{The category of Colombeau algebras}

In this section, we want to show that the Colombeau AG algebra, the related derivation of generalized functions and the embedding of distributions are all functorial constructions with respect to the change of AG. Although in this section we work on an arbitrary set of indices, we restrict our study only to embeddings of Schwartz distributions defined through a mollifier. Therefore, we are going to deal with mollifiers with null positive moments, namely with functions $\rho \in \mathcal{S}\left(\mathbb{R}^{n}\right)$ such that $\int \rho(x) x^{k} \mathrm{~d} x=0$ for every $k \in \mathbb{N}^{n},|k| \geq 1$. We call Colombeau mollifier any such function.

It is now worth recalling here why we are going to consider only embedding of Schwartz distributions based on a Colombeau mollifier and only principal AGs. On the one hand, Colombeau AG-algebras with $\mathcal{B}=\mathcal{Z}$, which are not necessarily of principal type, are important as a general framework. In fact, they include several examples studied in literature (see e.g. [6-11,15] and references therein). Moreover, this general abstract setting is crucial to understand where being a principal AG is a necessary property. For example, in [12, Thm. 4.12] we proved that

$$
f_{\varepsilon} *\left[b_{\varepsilon}^{n} \cdot \rho\left(b_{\varepsilon} \cdot-\right)\right]=f_{\varepsilon}+\mathcal{N}(\mathcal{Z}, \Omega)
$$

holds for all nets $\left(f_{\varepsilon}\right)$ of smooth functions compactly supported in $\Omega$ such that $\left(f_{\varepsilon}\right)$ is moderate with respect to $\mathrm{AG}(b)$, if and only if $b$ is a generator of $\mathcal{Z}$. Note that (8.1) is only slightly stronger than the usual universal property mentioned in (v) at Definition 40 , i.e.

$$
f *\left[b_{\varepsilon}^{n} \cdot \rho\left(b_{\varepsilon} \cdot-\right)\right]=f+\mathcal{N}(\mathcal{Z}, \Omega) \quad \forall f \in \mathcal{C}^{\infty}(\Omega)
$$

This result implies that there does not exist an embedding of Colombeau type (i.e. as in (8.1)) for non principal AGs. Summarizing, non-principal AGs are important because they include lots of interesting examples and because we need them to formulate results like [12, Thm. 4.12]. On the other hand, if we want to study embeddings of Colombeau type (8.1), we are forced to consider only principal AGs. It is also worth mentioning that a different type of embedding would still be possible. For example, using Zorn lemma and considering as $\Omega$ a convex subset, [25] shows that another interesting embedding is possible in Egorov algebras. We can therefore state that the 
existence of, maybe more involved, optimal embeddings of Schwartz distributions for non-principal AGs is still an open problem.

Definition 39 Let $n \in \mathbb{N}_{>0}$ be a fixed natural number. Then PAG denotes the category of principal $A G \mathrm{~s}$, whose objects are pairs $(b, \mathcal{B})$, where $\mathcal{B}$ is a principal $A G$ on a set of indices $\mathbb{I}$ and $b \in \mathcal{B}$ is a generator of $\mathcal{B}$. Arrows $f \in \operatorname{PAG}\left(\left(b_{1}, \mathcal{B}_{1}\right),\left(b_{2}, \mathcal{B}_{2}\right)\right)$ are morphisms $f \in \mathrm{AG}_{1}\left(\mathcal{B}_{1}, \mathcal{B}_{2}\right)$ of AGs that preserve the generator, i.e. such that $b_{1} \circ f=$ $b_{2}$. Let us note that if $f \in \operatorname{PAG}\left(\left(b_{1}, \mathcal{B}_{1}\right),\left(b_{2}, \mathcal{B}_{2}\right)\right)$ and $g \in \operatorname{PAG}\left(\left(b_{2}, \mathcal{B}_{2}\right),\left(b_{3}, \mathcal{B}_{3}\right)\right)$, then the composition in PAG is given as in $\mathrm{AG}_{1}$, i.e. by $f \circ g$ because $f: I_{2} \longrightarrow I_{1}$, $g: I_{3} \longrightarrow I_{2}$.

We observe that there exist non isomorphic pairs $\left(b, \mathbb{R}_{M}(b)\right),\left(c, \mathbb{R}_{M}(c)\right)$ defined on the set of indices $\mathbb{I}^{\mathrm{S}}$. In fact, let $b_{\varepsilon}=\varepsilon^{-1}$ and $c_{\varepsilon}=\left\lceil\varepsilon^{-1}\right\rceil$ for every $\varepsilon \in(0,1]$. Then $\left(c_{\varepsilon}\right)$ assumes only a countable amount of values, whilst $\left(b_{\varepsilon}\right)$ assumes a continuum of values. Hence, there can not be a morphism $f$ such that $b_{\varepsilon}=c_{f(\varepsilon)}$ for every $\varepsilon \in(0,1]$. The existence of non-isomorphic principal AGs also implies that the isomorphism stated in $[21,22,24]$ is not an isomorphism of principal AGs, i.e. it is not an arrow of the category $\mathrm{PAG}$.

Whilst the category PAG acts as domain in the Colombeau construction, the following category of Colombeau algebras acts as codomain.

Definition 40 Let DALG $_{\mathbb{R}}$ be the category of differential real algebras. We say that $(G, \partial, i) \in \mathrm{COL}_{n}$ if:

(i) $(G, \partial):\left(\mathcal{T} \mathbb{R}^{n}\right)^{\mathrm{op}} \longrightarrow \mathrm{DALG}_{\mathbb{R}}$ is a functor (i.e. it is a presheaf of differential real algebras). In particular, $\partial_{\Omega}^{\alpha}: G(\Omega) \longrightarrow G(\Omega)$ is a derivation for all $\alpha \in \mathbb{N}^{n}$.

(ii) If we think at both functors $\mathcal{D}^{\prime}, G:\left(\mathcal{T} \mathbb{R}^{n}\right)^{\text {op }} \longrightarrow \operatorname{VECT}_{\mathbb{R}}$ with values in the category of real vector spaces, then $\iota: \mathcal{D}^{\prime} \longrightarrow G$ is a natural transformation such that $\operatorname{ker}\left(\iota_{\Omega}\right)=\{0\}$ for every $\Omega \in \mathcal{T} \mathbb{R}^{n}$.

Moreover, for every $\Omega \in \mathcal{T} \mathbb{R}^{n}$, we have:

(iii) $\mathcal{C}^{\infty}(\Omega)$ is a subalgebra of $G(\Omega)$;

(iv) $\iota_{\Omega}\left(S_{\Omega}(f)\right)=f$ for all $f \in \mathcal{C}^{\infty}(\Omega)$;

(v) Let $D_{\Omega}^{\alpha}: \mathcal{D}^{\prime}(\Omega) \longrightarrow \mathcal{D}^{\prime}(\Omega)$ be the $\alpha \in \mathbb{N}^{n}$ derivation of distributions, then the following diagram commutes

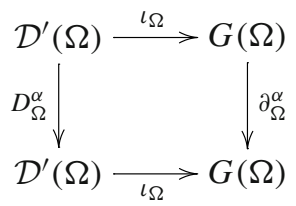

An arrow $\varphi \in \operatorname{Col}_{n}((G, \partial, \iota),(H, d, J))$ is a natural transformation $\varphi:(G, \partial) \longrightarrow$ $(H, d)$ such that for all $\Omega \in \mathcal{T} \mathbb{R}^{n}$ the following diagram commutes:

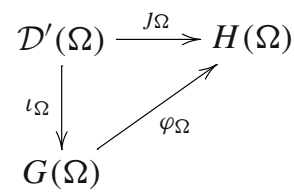


The following results prove the goal of the present section:

Lemma $41 \mathrm{COL}_{n}$ is a category.

Proof For every object $(G, \partial, \iota) \in \mathrm{CoL}_{n}$, the identity $1_{G(\Omega)}: G(\Omega) \longrightarrow G(\Omega)$ serves as the identity arrow of $(G, \partial, \iota)$ in $\mathrm{COL}_{n}$. To conclude the proof, it is sufficient to consider the composition of arrows. Let $\varphi \in \operatorname{COL}_{n}((G, \partial, \iota),(H, d, J))$, $\psi \in \operatorname{CoL}_{n}((H, d, J),(F, D, k))$, then $\psi \circ \varphi:(G, \partial) \longrightarrow(F, D)$ is a natural transformation and the following diagram commutes:

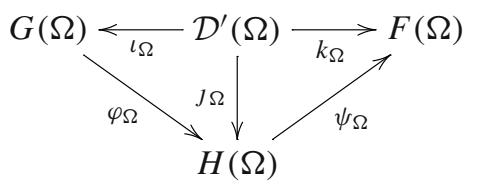

In particular, we have that

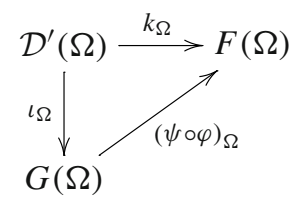

commutes, namely $\psi \circ \varphi \in \mathrm{COL}_{n}((G, \partial, \iota),(F, D, k))$.

Theorem 42 Let $\rho$ be a Colombeau mollifier. For each $(b, \mathcal{B}) \in \mathrm{PAG}$, set

$$
\operatorname{Co}_{n}^{\rho}(b, \mathcal{B}):=\left(\mathcal{G}(\mathcal{B},-), \partial, \iota_{b}^{\rho}\right)
$$

where $\iota_{b}^{\rho}$ is the usual embedding defined using the generator $b$ and the fixed mollifier $\rho$ (see [12, Sec. 4] for details). For $f \in \operatorname{PAG}\left(\left(b_{1}, \mathcal{B}_{1}\right),\left(b_{2}, \mathcal{B}_{2}\right)\right)$ and $\Omega \in \mathcal{T} \mathbb{R}^{n}$, set

$$
\operatorname{Co}_{n}^{\rho}(f)_{\Omega}:\left[u_{\varepsilon_{1}}\right] \in \mathcal{G}\left(\mathcal{B}_{1}, \Omega\right) \mapsto\left[u_{f\left(\varepsilon_{2}\right)}\right] \in \mathcal{G}\left(\mathcal{B}_{2}, \Omega\right)
$$

Then

$$
\operatorname{Co}_{n}^{\rho}: \mathrm{PAG} \longrightarrow \mathrm{COL}_{n}
$$

is a functor.

Proof The property $\operatorname{Co} \ell_{n}^{\rho}(b, \mathcal{B}) \in \operatorname{COL}_{n}$ for every $(b, \mathcal{B}) \in \mathrm{PAG}$ is a consequence of the results about Colombeau principal AG-algebras and embeddings of distributions proved in [12, Sec. 3 and 4].

We are left to prove the properties of $\mathcal{C o}_{n}^{\rho}$ with respect to arrows. First of all, let us prove that

$$
\begin{aligned}
\operatorname{Co}_{n}^{\rho}(f) & \in \operatorname{CoL}_{n}\left(\operatorname{Col}_{n}^{\rho}\left(b_{1}, \mathcal{B}_{1}\right), \operatorname{Co}_{n}^{\rho}\left(b_{2}, \mathcal{B}_{2}\right)\right) \\
& =\operatorname{CoL}_{n}\left(\left(\mathcal{G}\left(\mathcal{B}_{1},-\right), \partial, \iota_{b_{1}}^{\rho}\right),\left(\mathcal{G}\left(\mathcal{B}_{2},-\right), \partial, \iota_{b_{2}}^{\rho}\right)\right)
\end{aligned}
$$


for every $f \in \operatorname{PAG}\left(\left(b_{1}, \mathcal{B}_{1}\right),\left(b_{2}, \mathcal{B}_{2}\right)\right)$. Theorem 37 gives that $\operatorname{Co} \ell_{n}^{\rho}(f)_{\Omega}$ : $\mathcal{G}\left(\mathcal{B}_{1}, \Omega\right) \longrightarrow \mathcal{G}\left(\mathcal{B}_{2}, \Omega\right)$ is a morphism of $\mathbb{R}$-algebras (we recall that $\mathcal{G}(\mathcal{B}, \Omega):=$ $\mathcal{G}(\mathcal{B}, \mathcal{B}, \Omega)$ for every $\mathrm{AG} \mathcal{B})$. From $[12$, Thm. 4.7], it suffices to prove the commutativity of the diagram

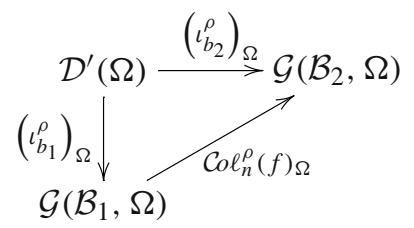

only for compactly supported $T \in \mathcal{D}^{\prime}(\Omega)$. In this case, we have

$$
\begin{aligned}
\operatorname{Co}_{n}^{\rho}(f)_{\Omega}\left[\left(\iota_{b_{1}}^{\rho}\right)_{\Omega}(T)\right] & =\operatorname{Co}_{n}^{\rho}(f)_{\Omega}\left[T * b_{1 \varepsilon_{1}} \odot \rho\right] \\
& =\left[T * b_{1 f\left(\varepsilon_{2}\right)} \odot \rho\right]=\left[T * b_{2 \varepsilon_{2}} \odot \rho\right]= \\
& =\left(\iota_{b_{2}}^{\rho}\right)_{\Omega}(T) .
\end{aligned}
$$

If $\Omega^{\prime} \subseteq \Omega$, then

$$
\begin{aligned}
\operatorname{Co}_{n}^{\rho}(f)_{\Omega^{\prime}}\left\{\left.\left[u_{\varepsilon_{1}}\right]\right|_{\Omega^{\prime}}\right\} & =\operatorname{Co} \ell_{n}^{\rho}(f)_{\Omega^{\prime}}\left[\left.u_{\varepsilon_{1}}\right|_{\Omega^{\prime}}\right] \\
& =\left[\left.u_{f\left(\varepsilon_{2}\right)}\right|_{\Omega^{\prime}}\right]=\left.\left\{\operatorname{Co}_{n}^{\rho}(f)_{\Omega}\left[u_{\varepsilon_{1}}\right]\right\}\right|_{\Omega^{\prime}} .
\end{aligned}
$$

This shows that $\operatorname{Co}_{n}^{\rho}(f): \mathcal{G}\left(\mathcal{B}_{1},-\right) \longrightarrow \mathcal{G}\left(\mathcal{B}_{2},-\right)$ is a natural transformation. To show (8.5), there remains to prove that $\operatorname{Co}_{n}^{\rho}(f)_{\Omega}$ is a morphism of differential algebras:

$$
\begin{aligned}
\operatorname{Co}_{n}^{\rho}(f)_{\Omega}\left\{\partial^{\alpha}\left[u_{\varepsilon_{1}}\right]\right\} & =\operatorname{Co} \ell_{n}^{\rho}(f)_{\Omega}\left[\partial u_{\varepsilon_{1}}^{\alpha}\right]= \\
& =\left[\partial^{\alpha} u_{f\left(\varepsilon_{2}\right)}\right]=\partial^{\alpha}\left\{\operatorname{Co}_{n}^{\rho}(f)_{\Omega}\left[u_{\varepsilon_{1}}\right]\right\} .
\end{aligned}
$$

Since $1_{I} \in \operatorname{PAG}((b, \mathcal{B}),(b, \mathcal{B}))$ is the identity in the category PAG, it is immediate to see that $\operatorname{Co} \ell_{n}^{\rho}\left(1_{I}\right)=1_{\mathcal{G}(\mathcal{B},-)}=1_{\mathcal{C}} \ell_{n}^{\rho}(b, \mathcal{B})$ from the definition of the map (8.4). Finally, let

$$
\begin{aligned}
& f \in \operatorname{PAG}\left(\left(b_{1}, \mathcal{B}_{1}\right),\left(b_{2}, \mathcal{B}_{2}\right)\right) \\
& g \in \operatorname{PAG}\left(\left(b_{2}, \mathcal{B}_{2}\right),\left(b_{3}, \mathcal{B}_{3}\right)\right) .
\end{aligned}
$$

Let $\left[u_{\varepsilon_{1}}\right] \in \mathcal{G}\left(\mathcal{B}_{1}, \Omega\right)$, then

$$
\begin{aligned}
\operatorname{Co}_{n}^{\rho}(g)_{\Omega}\left\{\operatorname{Co}_{n}^{\rho}(f)_{\Omega}\left(\left[u_{\varepsilon_{1}}\right]\right)\right\} & =\operatorname{Co}_{n}^{\rho}(g)_{\Omega}\left(\left[u_{f\left(\varepsilon_{2}\right)}\right]\right) \\
& =\left[u_{\left.f\left(g\left(\varepsilon_{3}\right)\right)\right]}\right]=\operatorname{Co}_{n}^{\rho}(f \circ g)_{\Omega}\left(\left[u_{\varepsilon_{1}}\right]\right) .
\end{aligned}
$$


Properties (i)-(v) of Definition 40 we started from have been taken as defining attributes for the category $\mathrm{COL}_{n}$ of Colombeau algebras. Although this gives an important role to these properties, it clearly does not aim to be an axiomatic characterization. For results in this direction see [21,22], where an axiomatic characterization is given but whose consistency depends on the generalized continuum hypothesis.

\section{Conclusions}

If we consider the most studied Colombeau algebra $\mathcal{G}^{s}(\Omega)=\mathcal{G}\left(\mathcal{B}_{\text {pol }}, \Omega\right)$ and if we have to deal with particular differential problems whose solutions grow more than polynomially in $\varepsilon$, then we are forced to consider a different algebra. Since our framework of Colombeau AG-algebras includes all known algebras of this type, this means that we are forced to consider a different AG. It is therefore natural to search for the correct notion of morphism of AGs, and to see whether Colombeau AG constructions behave in the correct way with respect to these morphisms. The results of Sects. 5, 7, 8 show that both the construction of the differential algebra and that of the embedding by means of a mollifier are functorial with respect to a natural notion of morphism of AGs. As shown in Sects. 6, 7, this permits to relate differential problems solved for different AGs.

Acknowledgements Open access funding provided by University of Vienna.

Open Access This article is distributed under the terms of the Creative Commons Attribution 4.0 International License (http://creativecommons.org/licenses/by/4.0/), which permits unrestricted use, distribution, and reproduction in any medium, provided you give appropriate credit to the original author(s) and the source, provide a link to the Creative Commons license, and indicate if changes were made.

\section{References}

1. Colombeau, J.F.: New Generalized Functions and Multiplication of Distributions. North-Holland, Amsterdam (1984)

2. Colombeau, J.F.: Elementary Introduction to New Generalized Functions. North-Holland, Amsterdam (1985)

3. Colombeau, J.F.: Multiplication of Distributions-A Tool in Mathematics, Numerical Engineering and Theoretical Physics. Springer, Berlin (1992)

4. Diener, M., Diener, F.: Ducks and Rivers: Three Existence Results. Nonstandard Analysis in Practice. Springer, Berlin (1995)

5. Grosser, M., Kunzinger, M., Oberguggenberger, M., Steinbauer, R.: Geometric Theory of Generalized Functions. Kluwer, Dordrecht (2001)

6. Delcroix, A.: Topology and functoriality in $(\mathcal{C}, \mathcal{E}, \mathcal{P})$-algebras. Application to singular differential problems. J. Math. Anal. Appl. 359, 394-403 (2009)

7. Delcroix, A., Pilipović, S., Hasler, M.F., Valmorin, V.: Sequence spaces with exponent weights. Realizations of Colombeau type algebras. Dissert. Math. 447, 1-73 (2007)

8. Delcroix, A., Scarpalezos, D.: Asymptotic scales-asymptotic algebras. Integral Transforms and Special Functions 6(1-4), 181-190 (1998)

9. Delcroix, A., Scarpalezos, D.: Topology on asymptotic algebras of generalized functions and applications. Monatsh. Math. 129, 1-14 (2000)

10. Egorov, Yu.V.: A contribution to the theory of generalized functions (Russian). Uspekhi Mat Nauk (Moskva) 45(5), 3-40 (1990). English translation in Russ. Math. Surveys 45(5), 1-49 (1990) 
11. Egorov, YuV: On generalized functions and linear differential equations Vestnik Moskov. Univ. Ser. I(2), 92-95 (1990). (Russian)

12. Giordano, P., Luperi Baglini, L.: Asymptotic gauges: generalization of Colombeau type algebras. Math. Nachr. 289(2-3), 247-274 (2016)

13. Giordano, P., Nigsch, E.: Unifying order structures for Colombeau algebras. Math. Nachr. 288(11-12), 1286-1302 (2015)

14. Hasler, M.F.: Asymptotic extension of topological modules and algebras. Integr. Transforms Spec. Funct. 20(3-4), 291-299 (2009)

15. Hasler, M.F.: On the relation between $(\mathcal{C}, \mathcal{E}, \mathcal{P})$-algebras and asymptotic algebras. Document de travail 2011-07. Centre d'Etude et de Recherche en Economie. Gestion, Modélisation et Informatique Appliquée (2011)

16. Lightstone, A.H., Robinson, A.: Nonarchimedean Fields and Asymptotic Expansions, vol. 13. NorthHolland, Amsterdam (1975)

17. Oberguggenberger, M., Todorov, T.: An embedding of Schwartz distributions in the algebra of asymptotic functions. Int. J. Math. Math. Sci. 21(3), 417-428 (1998)

18. Robinson, A.: Nonstandard Analysis. North Holland, Amsterdam (1966)

19. Robinson, A.: Function theory on some nonarchimedean fields. Am. Math. Mon. 80(6) (1973) (also in: Part II: Papers in the Foundations of Mathematics (1973), pp. 87-109)

20. Schwartz, L.: Sur 1' impossibilité de la multiplication des distributions. Comptes Rendus de L' Académie des Sciences 239, 847-848 (1954)

21. Todorov, T.D.: An axiomatic approach to the non-linear theory of generalized functions and consistency of Laplace transforms. Integr. Transforms Spec. Funct. 22(9), 695-708 (2011)

22. Todorov, T.D.: Algebraic approach to Colombeau theory. San Paulo J. Math. Sci. 7(2), 127-142 (2013)

23. Todorov, T.D., Vernaeve, H.: Full algebra of generalized functions and non-standard asymptotic analysis. Log. Anal. 1, 205-234 (2008)

24. Todorov, T.D., Wolf, R.: Hahn field representation of A. Robinsons asymptotic numbers. In: Delcroix, A., Hasler, M., Marti, J.A., Valmorin, V. (eds.) Nonlinear Algebraic Analysis and Applications, Proceedings of the International Conference on Generalized Functions (ICGF). Cambridge Scientific Publishers, Cottenham, pp. 357-374 (2004)

25. Vernaeve, H.: Optimal embeddings of distributions into algebras. Proc. Edinb. Math. Soc. 46, 373-378 (2003) 